\title{
Infrared and transcendental structure of two-loop supersymmetric QCD amplitudes
}

\author{
Gregor Kälin, ${ }^{a, b}$ Gustav Mogull, $^{a}$ Alexander Ochirov ${ }^{c}$ and Bram Verbeek ${ }^{a}$ \\ ${ }^{a}$ Department of Physics and Astronomy, Uppsala University, \\ Box 516, 75108 Uppsala, Sweden \\ ${ }^{b}$ SLAC National Accelerator Laboratory, Stanford University, \\ Stanford, CA 94309, U.S.A. \\ ${ }^{c}$ ETH Zürich, Institut für Theoretische Physik, \\ Wolfgang-Pauli-Str. 27, 8093 Zürich, Switzerland \\ E-mail: gregor.kaelin@physics.uu.se, gustav.mogull@physics.uu.se, \\ aochirov@phys.ethz.ch, bram.verbeek@physics.uu.se
}

ABSTRACT: Using a careful choice of infrared (IR) subtraction scheme, we demonstrate cancellation of all terms with transcendental weights $0,1,2$ from the finite part of the full-color two-loop four-gluon $\mathcal{N}=2$ supersymmetric QCD amplitude, with $N_{f}$ massless supersymmetric quarks. This generalizes the previously observed cancellation of weight2 terms in the superconformal theory, where $N_{f}=2 N_{c}$ for gauge group $\mathrm{SU}\left(N_{c}\right)$. The subtraction scheme follows naturally both from general IR factorization principles and from an integrand-level analysis of divergences in this amplitude. The divergences are written in terms of scalar triangle integrals whose expressions are known to all orders in the dimensional regulator $\epsilon=(4-D) / 2$. We also present integrated expressions for the full-color two-loop four-point amplitudes with both matter and vectors on external legs in which lower-weight terms also cancel using an appropriate IR scheme. This provides us with values for the two-loop cusp, gluonic, and quark anomalous dimensions in $\mathcal{N}=2$ supersymmetric QCD, which are cross-checked between the three different amplitudes.

KEYwords: Scattering Amplitudes, Supersymmetric Gauge Theory

ARXIV EPRINT: 1911.10218 


\section{Contents}

1 Introduction 1

1.1 Notation and conventions 3

2 IR factorization $\quad 5$

2.1 Soft-collinear exponentiation 5

2.2 Factorization for unrenormalized amplitudes 8

2.3 Subtracting IR structure of $\mathcal{N}=4$ SYM 9

2.4 Alternative IR subtraction 11

2.5 Divergence structure of $\mathcal{N}=2$ SQCD 12

3 IR and transcendental structure of one-loop $\mathcal{N}=2$ SQCD 14

$\begin{array}{lll}3.1 & \text { External vectors + matter } & 14\end{array}$

$\begin{array}{lll}3.2 & \text { External vectors } & 17\end{array}$

4 IR structure of two-loop $\mathcal{N}=2$ SQCD $\quad 19$

$\begin{array}{lll}4.1 & \text { External vectors } & 19\end{array}$

4.1.1 Planar and non-planar double boxes 21

$\begin{array}{lll}\text { 4.1.2 UV-divergent topologies } & 23\end{array}$

$\begin{array}{lll}4.2 & \text { External vectors + matter } & 25\end{array}$

5 Integration \& transcendental weight $\quad 26$

$\begin{array}{llr}6 & \text { Summary and outlook } & 28\end{array}$

A Anomalous dimensions in $\mathcal{N}=4$ SYM

B Two-loop finite remainder in $\mathcal{N}=2$ SQCD 33

\section{Introduction}

Infrared (IR) divergences are a vital aspect of the physics of scattering amplitudes in gauge theory, and in recent years our understanding of both has improved considerably [1-4]. In an amplitude with loop momenta $\left\{\ell_{i}\right\}$ there are two kinds of IR divergence: soft, where $\ell_{i} \rightarrow 0$, and collinear, where $\ell_{i} \rightarrow \tau p_{j}$ for an external momentum $p_{j}$. Most crucial has been the observation that for massless parton scattering, in the fixed-angle limit where all kinematic invariants $s_{i j}=2 p_{i} \cdot p_{j}$ are large, soft and collinear divergences factorize away from an IR-finite hard function [5,6]. The divergences take an exponential form involving an anomalous dimension [7-14], which at two loops justifies the form of the divergences predicted decades ago by Catani [15]. 
Despite this progress in understanding the IR behavior of multi-loop scattering amplitudes, modern computational methods often obscure IR structure, leading to it reappearing only after ultraviolet (UV) renormalization of final integrated results. To bridge this gap, efforts have been made to make the IR behavior apparent already at the integrand level. A notable example of this is the planar all-plus sector, where the IR structure - reduced in this case to the one-loop complexity - has been exploited to obtain compact two-loop integrands $[16,17]$ and amplitudes $[18-22]$ at five and higher points. In particular, the unrenormalized all-plus integrands were built from loop variables tailored to control IR divergences in specific regions.

Another important aspect of scattering amplitudes is their transcendental structure. Massless loop amplitudes can often be expressed in terms of multiple polylogarithms [23,24], the algebra of which is conjecturally graded by a property called transcendental weight. It corresponds to the number of integrations over rational kernels involved in a functions' definition: a logarithm (and $i \pi=\log (-1)$ ) has unit transcendental weight, a dilogarithm (and $\zeta_{2}$ ) has weight two, etc. $L$-loop amplitudes are observed to have an upper bound of weight $2 L$. In the case of amplitudes in the "simplest" gauge theory $[3,25]-\mathcal{N}=4$ supersymmetric Yang-Mills (SYM) - this bound has so far been observed to be saturated (see e.g. refs. [26-37]). This property is commonly referred to as maximal transcendentality $[38,39]$, and the maximally supersymmetric theory has thus far remained unique in this regard among Yang-Mills theories. The origin of this uniform-transcendentality property is not fully understood, and finding how and why it is violated in theories with $\mathcal{N}<4$ supersymmetries remains an open question.

In this paper, we further explore the connection between transcendental and IR structure in $\mathcal{N}=2$ supersymmetric theories initially observed in ref. [40]. In particular, we identify an IR subtraction that makes this connection maximally apparent. We approach the problem from two opposite sides. On the one hand, the two-loop IR divergence formulae, which follow from general factorization principles, are rewritten in section 2 so as to facilitate the analysis of our unrenormalized amplitudes. On the other hand, in sections 3 and 4 we examine the divergent parts of specific one- and two-loop amplitude integrands. Both analyses suggest a certain IR subtraction scheme in which divergences are written in terms of scalar triangle integrals, thereby extending the approach of refs. $[16,17]$ to a genuinely two-loop setting. In section 5 we verify that this scheme choice results in a cancellation of lower-weight terms from the finite, hard part of the two-loop four-gluon and two-gluon-two-matter amplitudes in $\mathcal{N}=2$ supersymmetric QCD (SQCD).

$\mathcal{N}=2$ SQCD consists of $\mathcal{N}=2$ supersymmetric Yang-Mills (SYM) theory coupled to $N_{f}$ massless supersymmetric quarks (hypermultiplets). As a model for quantum chromodynamics (QCD), the theory has significantly richer physics than the maximally constrained $\mathcal{N}=4 \mathrm{SYM}$. In particular, it has an arbitrary number of matter flavors $N_{f}$. For $N_{f}=C_{A} / T_{F}$ it develops a weakly-coupled superconformal phase, in which case its gluonic amplitudes become very similar to those in $\mathcal{N}=4 \mathrm{SYM}[40,41]$. The theory of $\mathcal{N}=2$ superconformal QCD (SCQCD), which is the aforementioned special case of $\mathcal{N}=2$ SQCD, has previously been seen to have interesting transcendental structure. Its planar four-point amplitudes were computed up through two loops in refs. [41-44]. Moreover, in 
ref. [40] integration of the full-color two-loop four-gluon amplitude in general $\mathcal{N}=2$ SQCD revealed that, for a particular choice of IR subtraction scheme in the superconformal theory, all terms with weight less than three cancel from the finite part of the amplitude. In this paper we extend this property to the full QCD-like theory using a judicious choice of IR subtraction scheme.

Achieving these results has been facilitated by remarkable properties of the $\mathcal{N}=2$ SQCD integrands obtained previously in refs. [45, 46]. In particular, diagrams containing internal matter lines are observed to diverge in fewer IR regions, as soft and collinear divergences arise only from virtual gluon exchanges. Purely gluonic diagrams (or those related to them by supersymmetry), which have the strongest IR divergences, are naturally eliminated from the remainder function $\mathcal{W}_{n}^{(L)}=\mathcal{M}_{n}^{(L)}-\mathcal{M}_{n}^{(L)[\mathcal{N}=4]}$ that measures scattering amplitudes as a "correction" to those in $\mathcal{N}=4$ SYM.

Finally, we also take this opportunity to complete the work begun in ref. [46] by presenting fully integrated two-loop four-point amplitudes with matter on external legs. The results provide us with expressions for the cusp, gluonic, and quark anomalous dimensions in $\mathcal{N}=2$ SQCD up to two-loop order, which are conveniently cross-checked by comparison between the different amplitudes.

\subsection{Notation and conventions}

Integration and normalizations. We use dimensional regularization in $D=4-2 \epsilon$ dimensions. Recurring conventional prefactors are

$$
S_{\epsilon}=(4 \pi)^{\epsilon} e^{-\epsilon \gamma_{\mathrm{E}}}, \quad r_{\Gamma}=e^{\epsilon \gamma_{\mathrm{E}}} \frac{\Gamma(1+\epsilon) \Gamma^{2}(1-\epsilon)}{\Gamma(1-2 \epsilon)}=1-\frac{1}{2} \zeta_{2} \epsilon^{2}-\frac{7}{3} \zeta_{3} \epsilon^{3}+\mathcal{O}\left(\epsilon^{4}\right),
$$

where $\gamma_{\mathrm{E}}=-\Gamma^{\prime}(1)$ is Euler's constant. Our $L$-loop integration operator I is accordingly normalized as

$$
\mathrm{I}[f(\ell)]=e^{\epsilon \gamma_{\mathrm{E}} L} \int \frac{\mathrm{d}^{D L} \ell}{\left(i \pi^{D / 2}\right)^{L}} \frac{f(\ell)}{D(f)},
$$

where $D(f)$ are the quadratic propagators associated with $f$. Unrenormalized (bare) $n$ point amplitudes $\mathcal{M}_{n}$ are expanded in powers of the bare coupling $\alpha_{\mathrm{s}}^{0}$ :

$$
\mathcal{M}_{n}=\left(4 \pi \alpha_{\mathrm{s}}^{0}\right)^{\frac{n-2}{2}} \sum_{L=0}^{\infty}\left(\frac{\alpha_{\mathrm{s}}^{0} S_{\epsilon}}{4 \pi}\right)^{L} \mathcal{M}_{n}^{(L)} .
$$

Color. The gauge group $G$ is arbitrary unless stated otherwise. We rely on a color operator $c$ which extracts the color factor of a given Feynman-like diagram expressed in terms of the structure constants $\tilde{f}^{a b c}$ and generators $T^{a}$ for gluonic and quark-gluon vertices, respectively,

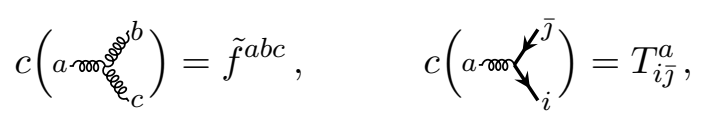

obeying the commutation relation $\left[T^{a}, T^{b}\right]_{i \bar{\jmath}}=\tilde{f}^{a b c} T_{i \bar{\jmath}}^{c}$. The Casimirs are defined as

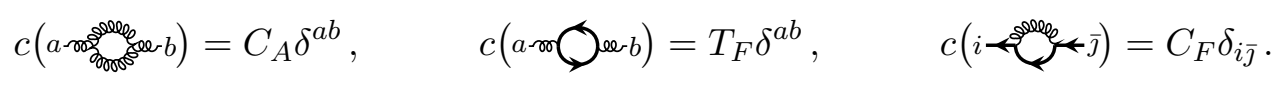


For $\mathrm{SU}\left(N_{c}\right)$ we normalize them as $C_{A}=2 N_{c}, T_{F}=1$, and $C_{F}=\left(N_{c}^{2}-1\right) / N_{c}$.

We also use the symbolic color generator $\mathbf{T}_{i}^{a}$ which belongs to the gauge-group representation of the $i$-th parton. For instance, $\left(\mathbf{T}^{a}\right)_{i \bar{\jmath}}=T_{i \bar{\jmath}}^{a},\left(\mathbf{T}^{a}\right)_{\bar{\imath} j}=-T_{j \bar{j}}^{a}$, and $\left(\mathbf{T}^{a}\right)_{b c}=\tilde{f}^{b a c}$ for external-state quarks, antiquarks, and gluons respectively. The dipole $\mathbf{T}_{i} \cdot \mathbf{T}_{j}$ is a conventional shorthand for the contraction $\sum_{a} \mathbf{T}_{i}^{a} \otimes \mathbf{T}_{j}^{a}$ over the adjoint indices - with the color indices of partons $i$ and $j$ still implicit and free.

Kinematics. All of our external momenta $p_{i}$ are taken outgoing. Kinematic invariants are denoted by $s_{i j}=2 p_{i} \cdot p_{j}$. At four points we use the Mandelstam variables $s=s_{12}$, $t=s_{23}$, and $u=s_{13}$. We split the $D$-dimensional loop momenta $\ell_{i}=\bar{\ell}_{i}+\ell^{[-2 \epsilon]}$ into four- and extra-dimensional parts, so that we can define the invariants $\mu_{i j}=-\ell_{i}^{[-2 \epsilon]} \cdot \ell_{j}^{[-2 \epsilon]}$. When $\ell_{i}$ and $p_{j}$ become collinear we write $\ell_{i} \| p_{j}$.

We often use Dirac traces to represent the kinematic dependence of amplitude numerators. They are defined via the spinor products (see e.g. ref. [4])

$$
\begin{aligned}
& {\left[i_{1} i_{2}\right]\left\langle i_{2} i_{3}\right\rangle \cdots\left[i_{k-1} i_{k}\right]\left\langle i_{k} i_{1}\right\rangle=p_{i_{1}}^{\mu_{1}} p_{i_{2}}^{\mu_{2}} \cdots p_{i_{k}}^{\mu_{k}} \operatorname{tr}\left(\bar{\sigma}_{\mu_{1}} \sigma_{\mu_{2}} \cdots \sigma_{\mu_{k}}\right)=\operatorname{tr}_{+}\left(i_{1} i_{2} \cdots i_{k}\right),} \\
& \left\langle i_{1} i_{2}\right\rangle\left[i_{2} i_{3}\right] \cdots\left\langle i_{k-1} i_{k}\right\rangle\left[i_{k} i_{1}\right]=p_{i_{1}}^{\mu_{1}} p_{i_{2}}^{\mu_{2}} \cdots p_{i_{k}}^{\mu_{k}} \operatorname{tr}\left(\sigma_{\mu_{1}} \bar{\sigma}_{\mu_{2}} \cdots \bar{\sigma}_{\mu_{k}}\right)=\operatorname{tr}_{-}\left(i_{1} i_{2} \cdots i_{k}\right),
\end{aligned}
$$

where $\sigma$ are the usual four-dimensional Pauli spin matrices. If an argument is a loop momentum $\ell_{i}$ it needs to be projected to its four-dimensional part $\bar{\ell}_{i}$.

Integrands. We present an $L$-loop full-color amplitude as a sum over a set $\Gamma_{n}^{(L)}$ of purely trivalent diagrams

$$
i \mathcal{M}_{n}^{(L)}=(-1)^{L} e^{\epsilon \gamma_{\mathrm{E}} L} \sum_{i \in \Gamma_{n}^{(L)}} \int \frac{\mathrm{d}^{L D} \ell}{\left(i \pi^{D / 2}\right)^{L}} \frac{\left(N_{f}\right)^{|i|}}{S_{i}} \frac{n_{i} c_{i}}{D_{i}}
$$

For each diagram we have

- a symmetry factor $S_{i}$;

- an overall factor $\left(N_{f}\right)^{|i|}$, where $|i|$ is the number of matter loops;

- a denominator $D_{i}$, absorbing the quadratic propagator denominators for the exposed internal edges of the diagram;

- a color factor $c_{i}$, corresponding to the color operator $c$ applied to the diagram;

- a numerator $n_{i}$, capturing all remaining kinematic dependence.

Different representations of the same integrand differ insofar as they assign different numerator factors to the diagrams. This way of organizing a full-color amplitude is tailored to exploit color-kinematics duality [47-51] and is alternative to the method of refs. [52-54]. 
State configurations. To organize the particle content of four-point MHV amplitudes in a QCD-like theory, we use $\kappa_{(i j)(k l)}$ introduced in refs. [46, 49] which carries the helicity weight of different external state configurations. It is defined to absorb the appropriate color-ordered tree amplitude as $i s t M_{4}^{(0)}(1,2,3,4)$ and is given by ${ }^{1}$

$$
\kappa_{(i j)(k l)}=\frac{[12][34]}{\langle 12\rangle\langle 34\rangle}\langle i j\rangle^{3}\langle k l\rangle,
$$

where $i<j$ and $k<l$. A particle label appearing in both parentheses of the subscript corresponds to a negative-helicity gluon $g^{-}$; a label not appearing at all corresponds to a positive-helicity gluon $g^{+}$; a label appearing only in the first parenthesis is a quark $q$; a label appearing in the second is an antiquark $\bar{q}$. When all external states are gluons, we also abbreviate

$$
\kappa_{i j} \equiv \kappa_{(i j)(i j)}
$$

For example, $\kappa_{12}=\kappa_{(12)(12)}$ corresponds to the state configuration $\left(g_{1}^{-}, g_{2}^{-}, g_{3}^{+}, g_{4}^{+}\right)$, and $\kappa_{(13)(14)}$ encodes $\left(g_{1}^{-}, g_{2}^{+}, q_{3}, \bar{q}_{4}\right)$. The use of symbolic $\kappa$-prefactors allows us to add amplitudes with different state configurations, which meshes well with their on-shell superspace interpretation in refs. [45, 46, 49].

\section{IR factorization}

In this section we review the IR factorization of UV-renormalized amplitudes in gauge theory and derive factorization formulae for their unrenormalized counterparts. The latter will be more useful for our subsequent analysis of $\mathcal{N}=2$ SQCD amplitudes.

\section{$2.1 \quad$ Soft-collinear exponentiation}

Through two loops the IR singularities of renormalized gauge theory amplitudes are entirely encoded by an anomalous dimension $[7-13]^{2}$

$$
\boldsymbol{\Gamma}_{\text {dip }}\left(\frac{p_{i}}{\mu}, \alpha_{\mathrm{s}}\right)=-\frac{\gamma_{K}\left(\alpha_{\mathrm{s}}\right)}{4} \sum_{i<j}^{n} \log \left(\frac{-s_{i j}}{\mu^{2}}\right) \mathbf{T}_{i} \cdot \mathbf{T}_{j}+\sum_{i=1}^{n} \gamma_{i}\left(\alpha_{\mathrm{s}}\right) .
$$

Apart from depending on the $n$-parton kinematic space, it involves a dipole operator $\mathbf{T}_{i} \cdot \mathbf{T}_{j}$ in the corresponding color space. That $\mathbf{T}_{i} \cdot \mathbf{T}_{j}$ only involves adjoint color in the intermediate state is important for later discussion of the IR finiteness of matter loop sub-diagrams. Moreover, it depends on the strong coupling constant exclusively through the (light-like) cusp anomalous dimension $\gamma_{K}$, as well as the field anomalous dimensions $\gamma_{i}$ of the partons which control hard collinear singularities. A renormalized amplitude $\widetilde{\mathcal{M}}_{n}$ factorizes as

$$
\widetilde{\mathcal{M}}_{n}\left(p_{i}, \mu, \alpha_{\mathrm{s}}(\mu)\right)=\mathbf{Z}\left(p_{i}, \mu, \alpha_{\mathrm{s}}(\mu)\right) \mathcal{H}_{n}\left(p_{i}, \mu, \alpha_{\mathrm{s}}(\mu)\right),
$$

\footnotetext{
${ }^{1}$ To obtain supersymmetric partners related by the Ward identities in $\mathcal{N}=1,2$ SQCD theories, one can promote $\langle i j\rangle^{\mathcal{N}}$ to $\delta^{2 \mathcal{N}}(Q)$, a supermomentum-conserving delta function, see e.g. ref. [2].

${ }^{2}$ Starting at three loops the complete anomalous dimension $\boldsymbol{\Gamma}\left(\frac{p_{i}}{\mu}, \alpha_{\mathrm{s}}\right)=\boldsymbol{\Gamma}_{\operatorname{dip}}\left(\frac{p_{i}}{\mu}, \alpha_{\mathrm{s}}\right)+\boldsymbol{\Delta}\left(\rho_{i j k l}, \alpha_{\mathrm{s}}\right)$ is corrected by a function $\boldsymbol{\Delta}$ of conformal-invariant cross ratios $\rho_{i j k l}=\frac{s_{i j} s_{k l}}{s_{i k} s_{j l}}$ [55-57], which has been confirmed by explicit calculation of the three-loop four-point $\mathcal{N}=4 \mathrm{SYM}$ amplitude [33].
} 
where the IR-divergent color-space operator ${ }^{3}$

$$
\mathbf{Z}\left(p_{i}, \mu, \alpha_{\mathrm{s}}(\mu)\right)=\exp \left\{-\int_{0}^{\mu} \frac{\mathrm{d} \lambda}{\lambda} \boldsymbol{\Gamma}_{\operatorname{dip}}\left(\frac{p_{i}}{\lambda}, \alpha_{\mathrm{s}}(\lambda)\right)\right\}
$$

acts on the hard-scattering amplitude $\mathcal{H}_{n}$, which is UV- and IR-finite by definition.

The IR poles in the dimensional-regularization parameter $\epsilon$ arise in $\mathbf{Z}$ from the integrated scale dependence of the strong coupling constant $\alpha_{\mathrm{s}}(\lambda)$. Let us see how this works at two loops, where only the first two orders in $\alpha_{\mathrm{s}}(\mu)$ are needed out of $\log \mathbf{Z}$. We expand all ingredients of the exponent in powers of the coupling constant:

$$
\gamma_{K}\left(\alpha_{\mathrm{s}}\right)=\sum_{L=1}^{\infty} \gamma_{K}^{(L)}\left(\frac{\alpha_{\mathrm{s}}}{2 \pi}\right)^{L}, \quad \gamma_{i}\left(\alpha_{\mathrm{s}}\right)=\sum_{L=1}^{\infty} \gamma_{i}^{(L)}\left(\frac{\alpha_{\mathrm{s}}}{2 \pi}\right)^{L} .
$$

To integrate the anomalous dimension, we need the scale dependence of the coupling constant $\alpha_{\mathrm{s}}(\lambda)$. It is convenient to expand it as power series in $\alpha_{\mathrm{s}}(\mu)$ :

$$
\alpha_{\mathrm{S}}(\lambda)=\alpha_{\mathrm{s}}(\mu)\left(\frac{\mu}{\lambda}\right)^{2 \epsilon} \sum_{l=0}^{\infty} a_{l}(\lambda, \mu)\left(\frac{\alpha_{\mathrm{s}}(\mu)}{2 \pi}\right)^{l}
$$

where the coefficient functions $a_{l}(\lambda, \mu)$ obey the initial conditions $a_{l}(\mu, \mu)=\delta_{0 l}$. The prefactor $(\mu / \lambda)^{2 \epsilon}$ is due to the leading term $-2 \epsilon \alpha_{\mathrm{s}}$ in the beta function,

$$
\beta\left(\alpha_{\mathrm{s}}\right)=-\alpha_{\mathrm{s}}\left\{2 \epsilon+\sum_{l=0}^{\infty} \beta_{l}\left(\frac{\alpha_{\mathrm{s}}}{2 \pi}\right)^{l+1}\right\}=-2 \epsilon \alpha_{\mathrm{s}}-\frac{\beta_{0}}{2 \pi} \alpha_{\mathrm{s}}^{2}+\mathcal{O}\left(\alpha_{\mathrm{s}}^{3}\right) .
$$

The renormalization group equation

$$
\beta\left(\alpha_{\mathrm{s}}(\lambda)\right)=\lambda \frac{\mathrm{d} \alpha_{\mathrm{s}}(\lambda)}{\mathrm{d} \lambda}=-2 \epsilon \alpha_{\mathrm{s}}(\lambda)+\alpha_{\mathrm{s}}(\mu)\left(\frac{\mu}{\lambda}\right)^{2 \epsilon} \sum_{l=0}^{\infty}\left(\frac{\alpha_{\mathrm{s}}(\mu)}{2 \pi}\right)^{l} \lambda \frac{\partial a_{l}(\lambda, \mu)}{\partial \lambda}
$$

then implies a system of linear differential equations that can be solved for $a_{l}(\lambda, \mu)$ :

$$
\left\{\begin{array} { r l } 
{ \lambda \frac { \partial a _ { 0 } ( \lambda , \mu ) } { \partial \lambda } } & { = 0 , } \\
{ \lambda \frac { \partial a _ { 1 } ( \lambda , \mu ) } { \partial \lambda } } & { = - \beta _ { 0 } ( \frac { \mu } { \lambda } ) ^ { 2 \epsilon } a _ { 0 } ^ { 2 } ( \lambda , \mu ) , } \\
{ \ldots } & { \Rightarrow }
\end{array} \quad \left\{\begin{array}{c}
a_{0}(\lambda, \mu)=1 \\
a_{1}(\lambda, \mu)=\frac{\beta_{0}}{2 \epsilon}\left[\left(\frac{\mu}{\lambda}\right)^{2 \epsilon}-1\right] \\
\ldots
\end{array}\right.\right.
$$

Therefore, the terms in eq. (2.5) that are relevant for the two-loop IR structure are

$$
\alpha_{\mathrm{s}}(\lambda)=\alpha_{\mathrm{s}}(\mu)\left(\frac{\mu}{\lambda}\right)^{2 \epsilon}\left\{1+\frac{\beta_{0} \alpha_{\mathrm{s}}(\mu)}{4 \pi \epsilon}\left[\left(\frac{\mu}{\lambda}\right)^{2 \epsilon}-1\right]\right\}+\mathcal{O}\left(\alpha_{\mathrm{s}}(\mu)^{3}\right) .
$$

\footnotetext{
${ }^{3}$ We omit the usual path-ordering sign as $\boldsymbol{\Gamma}_{\text {dip }}$ has only one non-trivial color operator and a constant that commutes with it.
} 
It is now straightforward to integrate the needed $\lambda$-dependent terms in the anomalous dimension. ${ }^{4}$ In this way, we obtain the IR poles encoded by $\mathbf{Z}$ in an explicit form:

$$
\begin{aligned}
& \log \mathbf{Z}= \frac{\alpha_{\mathrm{s}}(\mu)}{4 \pi}\left\{\frac{\gamma_{K}^{(1)}}{4} \sum_{i<j}^{n} \mathbf{T}_{i} \cdot \mathbf{T}_{j}\left[\frac{1}{\epsilon^{2}}-\frac{1}{\epsilon} \log \left(\frac{-s_{i j}}{\mu^{2}}\right)\right]+\frac{1}{\epsilon} \sum_{i=1}^{n} \gamma_{i}^{(1)}\right\} \\
&-\left(\frac{\alpha_{\mathrm{s}}(\mu)}{4 \pi}\right)^{2}\left\{\frac{1}{4} \sum_{i<j}^{n} \mathbf{T}_{i} \cdot \mathbf{T}_{j}\left[\frac{3 \beta_{0} \gamma_{K}^{(1)}}{4 \epsilon^{3}}-\frac{\gamma_{K}^{(2)}}{2 \epsilon^{2}}-\left(\frac{\beta_{0} \gamma_{K}^{(1)}}{2 \epsilon^{2}}-\frac{\gamma_{K}^{(2)}}{\epsilon}\right) \log \left(\frac{-s_{i j}}{\mu^{2}}\right)\right]\right. \\
&\left.+\sum_{i=1}^{n}\left(\frac{\beta_{0} \gamma_{i}^{(1)}}{2 \epsilon^{2}}-\frac{\gamma_{i}^{(2)}}{\epsilon}\right)\right\}+\mathcal{O}\left(\alpha_{\mathrm{s}}^{3}\right)
\end{aligned}
$$

To see how the IR divergences are organized perturbatively, we expand the complete and hard-scattering amplitudes in powers of the coupling:

$$
\widetilde{\mathcal{M}}_{n}=\left(4 \pi \alpha_{\mathrm{s}}\right)^{\frac{n-2}{2}} \sum_{L=0}^{\infty}\left(\frac{\alpha_{\mathrm{s}}}{4 \pi}\right)^{L} \widetilde{\mathcal{M}}_{n}^{(L)}, \quad \mathcal{H}_{n}=\left(4 \pi \alpha_{\mathrm{s}}\right)^{\frac{n-2}{2}} \sum_{L=0}^{\infty}\left(\frac{\alpha_{\mathrm{s}}}{4 \pi}\right)^{L} \mathcal{H}_{n}^{(L)} .
$$

We also introduce a convenient notation for the loop coefficients of $\log \mathbf{Z}$ :

$$
\log \mathbf{Z}=\frac{\alpha_{\mathrm{s}}(\mu)}{4 \pi} \mathbf{Y}^{(1)}(\epsilon)+\left(\frac{\alpha_{\mathrm{s}}(\mu)}{4 \pi}\right)^{2} \mathbf{Y}^{(2)}(\epsilon)+\mathcal{O}\left(\alpha_{\mathrm{s}}^{3}\right)
$$

Substituting these expansions into the factorization formula (2.2), we find

$$
\begin{aligned}
\widetilde{\mathcal{M}}_{n}^{(1)} & =\mathbf{Y}^{(1)}(\epsilon) \mathcal{M}_{n}^{(0)}+\mathcal{H}_{n}^{(1)}, \\
\widetilde{\mathcal{M}}_{n}^{(2)} & =\left[\mathbf{Y}^{(2)}(\epsilon)-\frac{1}{2}\left[\mathbf{Y}^{(1)}(\epsilon)\right]^{2}\right] \mathcal{M}_{n}^{(0)}+\mathbf{Y}^{(1)}(\epsilon) \widetilde{\mathcal{M}}_{n}^{(1)}+\mathcal{H}_{n}^{(2)},
\end{aligned}
$$

where $\mathcal{H}_{n}^{(0)}=\mathcal{M}_{n}^{(0)}$ (we drop the tilde as the tree amplitude is not renormalized). The IR operators $\mathbf{Y}^{(L)}(\epsilon)$ are then explicitly given as

$$
\begin{aligned}
& \mathbf{Y}^{(1)}(\epsilon)=\sum_{i<j}^{n} \mathbf{T}_{i} \cdot \mathbf{T}_{j}\left[\frac{1}{\epsilon^{2}}-\frac{1}{\epsilon} \log \left(\frac{-s_{i j}}{\mu^{2}}\right)\right]+\frac{1}{\epsilon} \sum_{i=1}^{n} \gamma_{i}^{(1)}, \\
& \mathbf{Y}^{(2)}(\epsilon)=-\frac{\beta_{0}}{\epsilon} \mathbf{Y}^{(1)}(\epsilon)+\left(\frac{\beta_{0}}{\epsilon}+\frac{\gamma_{K}^{(2)}}{2}\right) \mathbf{Y}^{(1)}(2 \epsilon)+\frac{1}{4 \epsilon} \sum_{i=1}^{n}\left(4 \gamma_{i}^{(2)}-\gamma_{K}^{(2)} \gamma_{i}^{(1)}\right) .
\end{aligned}
$$

We have plugged in the value $\gamma_{K}^{(1)}=4$ of the cusp anomalous dimension, which at the lowest order is regularization-scheme independent [58].

It is illuminating to compare these factorization formulae with the widely used expressions due to Catani and Seymour [15, 59]:

$$
\widetilde{\mathcal{M}}_{n}^{(1)}=\mathbf{I}^{(1)}(\epsilon) \mathcal{M}_{n}^{(0)}+\mathcal{O}\left(\epsilon^{0}\right), \quad \widetilde{\mathcal{M}}_{n}^{(2)}=\mathbf{I}^{(2)}(\epsilon) \mathcal{M}_{n}^{(0)}+\mathbf{I}^{(1)}(\epsilon) \widetilde{\mathcal{M}}_{n}^{(1)}+\mathcal{O}\left(\epsilon^{0}\right),
$$

${ }^{4}$ Two elementary integrals that are used in the derivation of eq. (2.10) are

$$
\int_{0}^{\mu} \frac{\mathrm{d} \lambda}{\lambda}\left(\frac{\mu}{\lambda}\right)^{2 k \epsilon}=-\frac{1}{2 k \epsilon}, \quad \int_{0}^{\mu} \frac{\mathrm{d} \lambda}{\lambda}\left(\frac{\mu}{\lambda}\right)^{2 k \epsilon} \log \left(\frac{-s}{\lambda^{2}}\right)=\frac{1}{2 k^{2} \epsilon^{2}}\left[1-k \epsilon \log \left(\frac{-s}{\mu^{2}}\right)\right] .
$$


in which the IR operators read $[7,60]$

$$
\begin{aligned}
\mathbf{I}^{(1)}(\epsilon)= & \frac{e^{\epsilon \gamma_{\mathrm{E}}}}{2 \Gamma(1-\epsilon)} \sum_{i=1}^{n}\left(\frac{1}{\epsilon^{2}}-\frac{2 \gamma_{i}^{(1)}}{\epsilon \mathbf{T}_{i}^{2}}\right) \sum_{j \neq i}^{n}\left(\frac{-s_{i j}}{\mu^{2}}\right)^{-\epsilon} \mathbf{T}_{i} \cdot \mathbf{T}_{j}=\mathbf{Y}^{(1)}(\epsilon)+\mathcal{O}\left(\epsilon^{0}\right), \\
\mathbf{I}^{(2)}(\epsilon)= & -\frac{1}{2} \mathbf{I}^{(1)}(\epsilon)\left(\mathbf{I}^{(1)}(\epsilon)+\frac{2 \beta_{0}}{\epsilon}\right)+\frac{e^{-\epsilon \gamma_{\mathrm{E}}} \Gamma(1-2 \epsilon)}{\Gamma(1-\epsilon)}\left(\frac{\beta_{0}}{\epsilon}+K_{\mathrm{R} . \mathrm{S} .}\right) \mathbf{I}^{(1)}(2 \epsilon) \\
& +\frac{e^{\epsilon \gamma_{\mathrm{E}}}}{4 \epsilon \Gamma(1-\epsilon)}\left[-\sum_{i=1}^{n} \frac{H_{i, \text { R.S. }}}{\mathbf{T}_{i}^{2}} \sum_{j \neq i}^{n}\left(\frac{-s_{i j}}{\mu^{2}}\right)^{-2 \epsilon} \mathbf{T}_{i} \cdot \mathbf{T}_{j}+\mathbf{H}_{\mathrm{R} . \mathrm{S} .}\right]
\end{aligned}
$$

The specific form of the operator $\mathbf{H}_{\mathrm{R} . \mathrm{S}}=\mathcal{O}\left(\epsilon^{0}\right)$ will be irrelevant to us, but we note that it is known to involve color structures of the form $\tilde{f}^{a b c} \mathbf{T}_{i}^{a} \mathbf{T}_{j}^{b} \mathbf{T}_{k}^{c}$ (see e.g. refs. [12, 61, 62]) that are absent from eq. (2.14). Moreover, notice that the $\epsilon$-dependence of $\mathbf{I}^{(L)}(\epsilon)$ is somewhat more involved compared to $\mathbf{Y}^{(L)}(\epsilon)$. The one-loop operators $\mathbf{I}^{(1)}(\epsilon)$ and $\mathbf{Y}^{(1)}(\epsilon)$ begin to differ after two orders in $\epsilon$. Although it is possible to identify

$$
K_{\text {R.S. }}=\frac{\gamma_{K}^{(2)}}{2}, \quad H_{i, \text { R.S. }}=4 \gamma_{i}^{(2)}-\gamma_{K}^{(2)} \gamma_{i}^{(1)}+\frac{3}{4} \beta_{0} \zeta_{2} \mathbf{T}_{i}^{2},
$$

this discrepancy makes the regularization-scheme dependence and color structure of $\mathbf{H}_{\mathrm{R} . \mathrm{S}}$. significantly more complicated than the explicit soft structure (2.10) warrants it $[8,9,12,13]$. Therefore, in the following we favor the latter and stick to the IR factorization formulae (2.13) and (2.14).

\subsection{Factorization for unrenormalized amplitudes}

We continue by studying the factorization properties fulfilled by unrenormalized amplitudes $\mathcal{M}_{n}^{(L)}$. Our definition of the beta function (2.6) is, in the $\overline{\mathrm{MS}}$ scheme, equivalent to the following relationship between bare and renormalized couplings:

$$
\alpha_{\mathrm{s}}^{0} S_{\epsilon}=\alpha_{\mathrm{s}} \mu^{2 \epsilon}\left\{1-\frac{\alpha_{\mathrm{s}} \beta_{0}}{4 \pi \epsilon}+\left(\frac{\alpha_{\mathrm{s}}}{4 \pi}\right)^{2}\left[\frac{\beta_{0}^{2}}{\epsilon^{2}}-\frac{\beta_{1}}{\epsilon}\right]+\mathcal{O}\left(\alpha_{\mathrm{s}}^{3}\right)\right\} .
$$

As we will not be needing the scale dependence anymore, for simplicity we have set $\mu=1$. This implies the usual renormalization relations

$$
\begin{aligned}
& \widetilde{\mathcal{M}}_{n}^{(1)}=\mathcal{M}_{n}^{(1)}-\frac{(n-2) \beta_{0}}{2 \epsilon} \mathcal{M}_{n}^{(0)}, \\
& \widetilde{\mathcal{M}}_{n}^{(2)}=\mathcal{M}_{n}^{(2)}-\frac{n \beta_{0}}{2 \epsilon} \mathcal{M}_{n}^{(1)}+\frac{(n-2)}{2}\left[\frac{n \beta_{0}^{2}}{4 \epsilon^{2}}-\frac{\beta_{1}}{\epsilon}\right] \mathcal{M}_{n}^{(0)} .
\end{aligned}
$$

Notice that the two-loop beta-function coefficient $\beta_{1}$, which is present in the UV renormalization above, does not appear in the IR divergence structure of renormalized amplitudes until three-loop order [8, 9, 55, 63].

Combining eqs. (2.13a) and (2.19a) at one loop is simple:

$$
\mathcal{M}_{n}^{(1)}=\widetilde{\mathcal{M}}_{n}^{(1)}+\frac{(n-2) \beta_{0}}{2 \epsilon} \mathcal{M}_{n}^{(0)}=\left[\mathbf{Y}^{(1)}(\epsilon)+\frac{(n-2) \beta_{0}}{2 \epsilon}\right] \mathcal{M}_{n}^{(0)}+\mathcal{H}_{n}^{(1)}
$$


A similar exercise at two loops is more cumbersome but straightforward, and we present the answer below. In order to make the pole structure of the amplitude more transparent, we separate the one-loop IR operator $\mathbf{Y}^{(1)}(\epsilon)$ into its $1 / \epsilon$ monopole (collinear) and $1 / \epsilon^{2}$ dipole (soft) parts:

$$
\mathbf{Y}^{(1)}(\epsilon)=\mathbf{S}(\epsilon)+\frac{1}{\epsilon} \sum_{i=1}^{n} \gamma_{i}^{(1)}, \quad \mathbf{S}(\epsilon)=\frac{1}{\epsilon^{2}} \sum_{i<j}^{n} \mathbf{T}_{i} \cdot \mathbf{T}_{j}\left[1-\epsilon \log \left(-s_{i j}\right)\right] .
$$

In terms of this dipole operator $\mathbf{S}(\epsilon)$, we find that the unrenormalized amplitudes factorize as

$$
\begin{aligned}
\mathcal{M}_{n}^{(1)}=\mathbf{S}(\epsilon) \mathcal{M}_{n}^{(0)} & +\frac{1}{\epsilon}\left[\frac{n-2}{2} \beta_{0}+\sum_{i=1}^{n} \gamma_{i}^{(1)}\right] \mathcal{M}_{n}^{(0)}+\mathcal{H}_{n}^{(1)} \\
\mathcal{M}_{n}^{(2)}=\mathbf{S}(\epsilon) \mathcal{M}_{n}^{(1)} & -\frac{1}{2} \mathbf{S}(\epsilon) \mathbf{S}(\epsilon) \mathcal{M}_{n}^{(0)}+\left[\frac{\beta_{0}}{\epsilon}+\frac{\gamma_{K}^{(2)}}{2}\right] \mathbf{S}(2 \epsilon) \mathcal{M}_{n}^{(0)} \\
& +\frac{1}{2 \epsilon^{2}}\left[\frac{n-2}{2} \beta_{0}+\sum_{i=1}^{n} \gamma_{i}^{(1)}\right]\left[\frac{n}{2} \beta_{0}+\sum_{i=1}^{n} \gamma_{i}^{(1)}\right] \mathcal{M}_{n}^{(0)} \\
& +\frac{1}{\epsilon}\left[\frac{n-2}{2} \beta_{1}+\sum_{i=1}^{n} \gamma_{i}^{(2)}\right] \mathcal{M}_{n}^{(0)}+\frac{1}{\epsilon}\left[\frac{n}{2} \beta_{0}+\sum_{i=1}^{n} \gamma_{i}^{(1)}\right] \mathcal{H}_{n}^{(1)}+\mathcal{H}_{n}^{(2)}
\end{aligned}
$$

These formulae capture all $\epsilon$-divergences in dimensional regularization through two loops. They hold in a general gauge theory with massless matter, since at this point we have not specialized to $\mathcal{N}=2$ SQCD in any way.

The above factorization formulae contain dependence on the scheme of dimensional regularization [64-68] (see ref. [69] for a recent overview), which is hardly surprising. In fact, the beta-function coefficients $\beta_{L-1}$ and the anomalous dimensions $\gamma_{K}^{(L)}$ and $\gamma_{i}^{(L)}$ may also depend on the specific subtraction scheme used for removing the UV divergences [58, 70]. It is, however, clear that the latter dependence should be spurious in the factorization properties (2.22) of unrenormalized amplitudes. ${ }^{5}$

\subsection{Subtracting IR structure of $\mathcal{N}=4 \mathrm{SYM}$}

In this section we specialize to the case where all external partons are gluons (or more generally vector multiplets that include gluons) and define the discrepancy between the $n$-gluon amplitude in question and its $\mathcal{N}=4$ counterpart. The remainder function has a simpler divergence structure than the original amplitude.

Due to a simple relation between the gluonic collinear anomalous dimension and the $\beta$-function coefficient (see e.g. ref. [58]),

$$
\gamma_{g}^{(1)}=-\frac{\beta_{0}}{2}
$$

\footnotetext{
${ }^{5}$ For a non-supersymmetric theory considered in the dimensional-reduction (DRED [66]) or the fourdimensional-helicity (FDH [67, 68]) scheme, eq. (2.22) would need to take into account a set of additional evanescent scalars with two separate running couplings $\alpha_{4 \epsilon}$ and $\alpha_{\mathrm{e}}$ for their self- and gauge interactions [71-73]. However, the principal purpose of these schemes is to preserve supersymmetry, which pegs the evanescent couplings to the gauge coupling. So in $\mathcal{N}=2$ SQCD we can use the precise formulae (2.22) in a close analogue of the FDH scheme, which we will discuss in section 2.5.
} 
the factorization formulae (2.22) for purely gluonic amplitudes can be rewritten in a streamlined form:

$$
\begin{aligned}
& \mathcal{M}_{n}^{(1)}=\mathbf{S}(\epsilon) \mathcal{M}_{n}^{(0)}-\frac{\beta_{0}}{\epsilon} \mathcal{M}_{n}^{(0)}+\mathcal{H}_{n}^{(1)}, \\
& \mathcal{M}_{n}^{(2)}=\mathbf{S}(\epsilon) \mathcal{M}_{n}^{(1)}+\left\{-\frac{1}{2} \mathbf{S}(\epsilon) \mathbf{S}(\epsilon)+\left[\frac{\beta_{0}}{\epsilon}+\frac{\gamma_{K}^{(2)}}{2}\right] \mathbf{S}(2 \epsilon)+\frac{1}{\epsilon}\left[\frac{n-2}{2} \beta_{1}+n \gamma_{g}^{(2)}\right]\right\} \mathcal{M}_{n}^{(0)}+\mathcal{H}_{n}^{(2)} .
\end{aligned}
$$

Let us turn for a moment to the special case of $\mathcal{N}=4$ SYM. Its gluonic tree-level amplitudes $\mathcal{M}_{n}^{(0)}$ trivially coincide with those in massless QCD (or any four-dimensional gauge theory for that matter); the specifics of the matter content start to enter at one loop. Moreover, the theory is UV finite (hence $\beta_{i}=0$ ) and only has IR divergences. Despite being considered in many ways the simplest gauge theory [25], its scattering amplitudes contain the most IR-divergent kinematic regions. The above formulae simplify to

$$
\begin{aligned}
& \mathcal{M}_{n}^{(1)[\mathcal{N}=4]}=\mathbf{S}(\epsilon) \mathcal{M}_{n}^{(0)}+\mathcal{H}_{n}^{(1)[\mathcal{N}=4]} \\
& \mathcal{M}_{n}^{(2)[\mathcal{N}=4]}=\mathbf{S}(\epsilon) \mathcal{M}_{n}^{(1)[\mathcal{N}=4]}+\left\{-\frac{1}{2} \mathbf{S}(\epsilon) \mathbf{S}(\epsilon)+\frac{1}{2} \gamma_{K}^{(2)[\mathcal{N}=4]} \mathbf{S}(2 \epsilon)+\frac{n}{\epsilon} \gamma_{g}^{(2)[\mathcal{N}=4]}\right\} \mathcal{M}_{n}^{(0)}+\mathcal{H}_{n}^{(2)[\mathcal{N}=4]},
\end{aligned}
$$

where the anomalous dimensions are $[60,63,74,75]$

$$
\gamma_{K}^{(2)[\mathcal{N}=4]}=-2 \zeta_{2} C_{A}, \quad \gamma_{g}^{(2)[\mathcal{N}=4]}=\frac{1}{8} \zeta_{3} C_{A}^{2} .
$$

These formulae are consistent with the iterative construction of refs. [27, 28], as verified in appendix A.

We wish to study the remainder function $\mathcal{W}_{n}^{(L)}$, which we define perturbatively as the difference between the (gluonic) amplitude in an arbitrary gauge theory and that of $\mathcal{N}=4$ SYM:

$$
\mathcal{M}_{n}^{(L)}=\mathcal{M}_{n}^{(L)[\mathcal{N}=4]}+\mathcal{W}_{n}^{(L)} .
$$

Assuming the factorization (2.25) for the amplitudes in $\mathcal{N}=4 \mathrm{SYM}$, we find that these subtracted amplitudes factorize in a much simpler way:

$$
\begin{aligned}
\mathcal{W}_{n}^{(1)}=-\frac{\beta_{0}}{\epsilon} \mathcal{M}_{n}^{(0)} & +\mathcal{H}_{n}^{(1)}-\mathcal{H}_{n}^{(1)[\mathcal{N}=4]} \\
\mathcal{W}_{n}^{(2)}=\mathbf{S}(\epsilon) \mathcal{W}_{n}^{(1)} & +\left(\frac{\beta_{0}}{\epsilon}+\frac{1}{2}\left[\gamma_{K}^{(2)}-\gamma_{K}^{(2)[\mathcal{N}=4]}\right]\right) \mathbf{S}(2 \epsilon) \mathcal{M}_{n}^{(0)} \\
& +\frac{1}{\epsilon}\left(\frac{n-2}{2} \beta_{1}+n\left[\gamma_{g}^{(2)}-\gamma_{g}^{(2)[\mathcal{N}=4]}\right]\right) \mathcal{M}_{n}^{(0)}+\mathcal{H}_{n}^{(2)}-\mathcal{H}_{n}^{(2)[\mathcal{N}=4]}
\end{aligned}
$$

where the hierarchy of $1 / \epsilon^{k}$ poles is maximally transparent. Namely,

- The most singular $1 / \epsilon^{2 L}$ amplitude pieces are absent from eq. (2.28) as they have been absorbed by the $\mathcal{N}=4$ amplitudes.

- The presence of $\beta_{0}$ in front of the $1 / \epsilon$ terms in eq. (2.28a) suggests that the remaining one-loop divergences are exclusively UV, i.e. the complete IR structure of purely gluonic amplitudes is at one loop captured by $\mathcal{N}=4 \mathrm{SYM}$. 
- The leading $1 / \epsilon^{3}$ amplitude pieces in eq. (2.28b) are also accompanied by $\beta_{0}$. Therefore, they occur due to the multiplication of the UV divergence $1 / \epsilon$ in one of the two loops by the IR divergences $1 / \epsilon^{2}$ in the other.

- All of the $1 / \epsilon^{2}$ terms in $\mathcal{W}_{n}^{(2)}$ are caused by the overlap of soft and collinear divergences in only one of the two loops, while the other loop stays finite.

- The remaining $1 / \epsilon$ terms arise due to a single type of UV, soft or collinear divergence occurring in one of the loops.

The emergence of this divergence hierarchy from the one-loop integrands in $\mathcal{N}=2$ SQCD is discussed in more detail in section 3, and the two-loop integrands in section 4 .

\subsection{Alternative IR subtraction}

In anticipation of specializing to $\mathcal{N}=2$ SQCD (but not doing so yet) we find it convenient to rewrite eq. (2.28b) in terms of scalar triangle integrals. ${ }^{6}$ For instance, the one-loop dipole operator (2.21) may be represented by

$$
\mathbf{S}(\epsilon)=\sum_{i<j}^{n} s_{i j}=\mathbf{T}_{i}^{i} \cdot \mathbf{T}_{j}+\mathcal{O}\left(\epsilon^{0}\right)
$$

where the missing higher-order terms come from one-loop triangle integral:

$$
\chi_{j}^{i}=-\frac{r_{\Gamma}}{\epsilon^{2}}\left(-s_{i j}\right)^{-1-\epsilon}=\frac{1}{\epsilon^{2} s_{i j}}\left[1-\epsilon \log \left(-s_{i j}\right)+\frac{\epsilon^{2}}{2}\left(\log ^{2}\left(-s_{i j}\right)-\zeta_{2}\right)\right]+\mathcal{O}(\epsilon) .
$$

Similarly, using the two-loop triangle integrals (see e.g. ref. [76])

$$
\begin{aligned}
& =\oint_{j}^{i}=-\frac{r_{\Gamma} e^{\epsilon \gamma_{\mathrm{E}}} \Gamma^{2}(1-2 \epsilon) \Gamma(1+2 \epsilon)}{4 \epsilon^{3}(1-2 \epsilon) \Gamma(1-3 \epsilon)}\left(-s_{i j}\right)^{-1-2 \epsilon}, \\
& =\mathcal{g}_{j}^{i}=-\frac{e^{2 \epsilon \gamma_{\mathrm{E}}} \Gamma(1+2 \epsilon) \Gamma^{3}(1-\epsilon)}{2 \epsilon^{3}(1-2 \epsilon) \Gamma(1-3 \epsilon)}\left(-s_{i j}\right)^{-1-2 \epsilon},
\end{aligned}
$$

the two-loop factorization formula (2.28b) may be non-trivially rearranged into

$$
\begin{aligned}
\mathcal{W}_{n}^{(2)}= & \left(\sum_{i<j}^{n} s_{i j}=\mathcal{Y}_{j}^{i} \mathbf{T}_{i} \cdot \mathbf{T}_{j}\right) \mathcal{W}_{n}^{(1)}+\beta_{0}\left(\sum_{i<j}^{n} s_{i j}\left[\boldsymbol{q}_{j}^{i}-\dot{\phi}_{j}^{i}\right] \mathbf{T}_{i} \cdot \mathbf{T}_{j}\right) \mathcal{M}_{n}^{(0)} \\
& -2\left(\beta_{0}-\frac{1}{4}\left[\gamma_{K}^{(2)}-\gamma_{K}^{(2)[\mathcal{N}=4]}\right]\right) \mathbf{S}(2 \epsilon) \mathcal{M}_{n}^{(0)} \\
& +\frac{1}{\epsilon}\left(\frac{n}{8}\left(4-\zeta_{2}\right) \beta_{0} C_{A}+\frac{n-2}{2} \beta_{1}+n\left[\gamma_{g}^{(2)}-\gamma_{g}^{(2)[\mathcal{N}=4]}\right]\right) \mathcal{M}_{n}^{(0)}+\mathcal{W}_{n}^{(2) \mathrm{fin}}
\end{aligned}
$$

To verify this, one requires color conservation $\sum_{i} \mathbf{T}_{i}=0$, which for external gluons implies $\sum_{i, j \neq i}^{n} \mathbf{T}_{i} \cdot \mathbf{T}_{j}=-n C_{A}$. Moreover, finite $\mathcal{O}\left(\epsilon^{0}\right)$ terms have been absorbed into the hard

\footnotetext{
${ }^{6}$ Rewriting IR operators in terms of scalar triangles has already proved fruitful in refs. [16, 52].
} 


\begin{tabular}{|c|c|c|c|c|}
\hline hel. th & $\mathcal{N}=4 \mathrm{SYM}$ & \multicolumn{2}{|c|}{$\mathcal{N}=2 \mathrm{SQCD}$} & QCD \\
\hline+1 & 1 & 1 & $\times N_{f}$ & $1 \times N_{f}$ \\
\hline$+1 / 2$ & 4 & 2 & 11 & 11 \\
\hline 0 & 6 & $\begin{array}{ll}1 & 1\end{array}$ & 22 & \\
\hline$-1 / 2$ & 4 & 2 & 11 & 1 \\
\hline-1 & 1 & 1 & & 1 \\
\hline rep. & adj. & adj. & 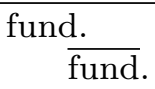 & 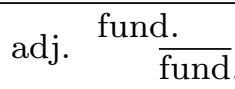 \\
\hline
\end{tabular}

Table 1. On-shell states of $\mathcal{N}=2 \mathrm{SQCD}$ in comparison with $\mathcal{N}=4 \mathrm{SYM}$ and conventional massless QCD. The helicities are listed in the left column, and $\mathrm{SU}\left(N_{c}\right)$-group representations of the particles are shown in the lower row.

function, so $\mathcal{W}_{n}^{(2) \text { fin }} \neq \mathcal{H}_{n}^{(2)}-\mathcal{H}_{n}^{(2)[\mathcal{N}=4]}$. We have therefore mandated a new IR subtraction scheme for $\mathcal{W}_{n}^{(2)}$ which differs from the minimal scheme given in eq. (2.28b). As we shall see in the next subsection, when the two-loop anomalous dimensions $\gamma_{K}^{(2)}$ and $\gamma_{g}^{(2)}$ for $\mathcal{N}=2$ SQCD are inserted, the second two lines in eq. (2.32) cancel away.

\subsection{Divergence structure of $\mathcal{N}=2$ SQCD}

We discuss in more detail the $\mathcal{N}=2$ SQCD theory, whose amplitudes we study in this paper. The theory is built from $\mathcal{N}=2 \mathrm{SYM}$ coupled to $N_{f}$ supersymmetric quarks transforming under the fundamental representation of the gauge group $G$. One can view it as a two-fold supersymmetry enhancement of QCD, or as an orbifold truncation of $\mathcal{N}=4$ SYM [77-81], where half of the particle content is promoted to the fundamental or antifundamental representation of the gauge group [45]. In table 1 we summarize its particle-helicity content while displaying its intermediate position between the physical theory of massless QCD and the maximally supersymmetric $\mathcal{N}=4$ SYM theory (in which no matter content is permitted).

The intermediate complexity of $\mathcal{N}=2$ SQCD is reflected in its UV structure. Recall that the one-loop beta-function coefficient for a gauge theory minimally coupled to $n_{f}$ Weyl fermions and $n_{s}$ real scalars is

$$
\beta_{0}=\frac{11}{6} C_{A}-\frac{1}{3} T_{f} n_{f}-\frac{1}{12} T_{s} n_{s} .
$$

$\mathcal{N}=2$ SYM theories with matter are known to have a one-loop exact beta-function $[82,83]$, so for $\mathcal{N}=2 \mathrm{SQCD}$ we get

$$
\beta\left(\alpha_{\mathrm{s}}\right)=-\alpha_{\mathrm{s}}\left(2 \epsilon+\beta_{0} \frac{\alpha_{\mathrm{s}}}{2 \pi}\right), \quad \beta_{0}=C_{A}-T_{F} N_{f} \stackrel{\mathrm{SU}\left(N_{c}\right)}{=} 2 N_{c}-N_{f} .
$$

This $\beta_{0}$ value can be computed from the general formula (2.33) by substituting $n_{f}=n_{s}=2, T_{f}=T_{s}=C_{A}$ for the adjoint fermions and scalars and $n_{f}=2 N_{f}, n_{s}=4 N_{f}$, 
$T_{f}=T_{s}=T_{F}$ for (the parity-even combination of) the fundamental and antifundamental matter multiplets.

As is often done in QCD, we leave the number of matter flavors $N_{f}$ arbitrary. The notable special cases are the pure $\mathcal{N}=2 \mathrm{SYM}$ theory for $N_{f}=0$ and the $\mathcal{N}=2$ superconformal (SCQCD) theory for $N_{f}=C_{A} / T_{F}$ [83]. Moreover, it is evident from table 1 that if we switch the representation of one of the matter multiplets to adjoint, in combination with the $\mathcal{N}=2$ vector multiplet it will enhance it to the $\mathcal{N}=4$ one. This allows us to impose certain $\mathcal{N}=4$ matching conditions $[45,46]$ on the kinematic numerators (illustrated by eqs. (3.12) and (4.3) below) which will significantly facilitate our integrand-level analysis of the two-loop IR structure in section 4.

Like $\mathcal{N}=4 \mathrm{SYM}$, the $\mathcal{N}=2$ SCQCD theory is UV finite. However, it also contains matter amplitudes with a non-trivial IR divergence structure. Therefore, if we expand $\mathcal{N}=2 \mathrm{SQCD}$ theory around the conformal fixed point $N_{f}=C_{A} / T_{F}$, we observe a clean separation between different kinds of divergences. By explicit calculation of three different two-loop amplitudes (to be expanded upon in section 5) we find that the cusp and collinear anomalous dimensions can be written as

$$
\begin{array}{lll}
\gamma_{K}^{(1)}=4, & \gamma_{K}^{(2)}=-2 \zeta_{2} C_{A}+4 \beta_{0}, \\
\gamma_{g}^{(1)}=-\frac{\beta_{0}}{2}, & \gamma_{g}^{(2)}=\frac{1}{8} C_{A}\left(\zeta_{3} C_{A}+\beta_{0}\left(\zeta_{2}-4\right)\right), \\
\gamma_{q}^{(1)}=\gamma_{\bar{q}}^{(1)}=0, & \gamma_{q}^{(2)}=\gamma_{\bar{q}}^{(2)}=\frac{1}{8} C_{F}\left(13 \zeta_{3} C_{A}-12 \zeta_{3} C_{F}-\beta_{0}\left(3 \zeta_{2}+4\right)\right) .
\end{array}
$$

When $\beta_{0}=0$ both $\gamma_{K}^{(2)}$ and $\gamma_{g}^{(2)}$ coincide with their $\mathcal{N}=4$ values given in eq. (2.26). This is consistent with the known observation that cusp anomalous dimensions in $\mathcal{N}=2$ SCQCD and $\mathcal{N}=4 \mathrm{SYM}$ only start to differ at three loops [84-86]. If we furthermore promote the SCQCD matter to the adjoint representation (by substituting $T_{F}, C_{F} \rightarrow C_{A}$ ) it becomes a part of the larger $\mathcal{N}=4$ multiplet, such that $\gamma_{q}^{(2)}$ coincides with $\gamma_{g}^{(2)[\mathcal{N}=4]}$. The same identification happens for the gauge group $G=\mathrm{SO}(3)$, in which case $C_{A}=N_{c}-2=1$ and $C_{F}=\frac{1}{2}\left(N_{c}-1\right)=1-$ setting $\beta_{0}=0$ gives $N_{f}=C_{A} / T_{F}=1$, and we recover the $\mathcal{N}=4$ SYM theory.

We invite the reader to confirm the cancellation of the second two lines in eq. (2.32), which occurs when the specific values of $\gamma_{K}^{(2)}$ and $\gamma_{g}^{(2)}$ quoted above are inserted. Soft, collinear, and UV divergences are now all exposed: soft occurring between massless legs of the triangle integrals, collinear at their vertices, and UV in the embedded bubble integrals. Notice the placement of $\beta_{0}$ : since $\mathcal{W}_{n}^{(1)}=-\frac{\beta_{0}}{\epsilon} \mathcal{M}_{n}^{(0)}+\mathcal{O}\left(\epsilon^{0}\right)$, setting $\beta_{0}=0$ manifestly eliminates all UV divergences, and $\mathcal{W}_{n}^{(2)}$ diverges only as $\mathcal{O}\left(\epsilon^{-2}\right)$. As we shall see in section 5 , this new scheme is privileged as it succeeds in cancelling all terms from $\mathcal{W}_{4}^{(2) \text { fin }}$ with transcendental weight less than three. To better motivate eq. (2.32) we will examine the IR structure of the two-loop four-gluon integrand in section 4, which exhibits the divergence factorization structure in a surprisingly transparent way.

Our one- and two-loop integrands are obtained by matching to generalized unitarity cuts [87-90]. Extra-dimensional terms $\mu_{i j}$, required by dimensional regularization, are obtained by evaluating six-dimensional unitarity cuts $[91,92]$ for chiral $\mathcal{N}=(1,0) \mathrm{SYM}$ 
coupled to $N_{f}$ copies of the $\mathcal{N}=(0,1)$ hypermultiplet. ${ }^{7}$ As explained in ref. [45] (see also refs. [93-97]), this theory is a natural six-dimensional uplift of $\mathcal{N}=2$ SQCD. It defines our dimensional regularization scheme: external states are strictly four-dimensional (like in the 't Hooft-Veltman scheme [64]), and the internal state counting precisely matches that of the four-dimensional $\mathcal{N}=2$ SQCD theory. This is therefore a close analogue of the four-dimensional helicity (FDH $[67,68]$ ) scheme. The anomalous dimensions $(2.35)$ are therefore consistent with their FDH values [58, 61, 62, 98].

\section{IR and transcendental structure of one-loop $\mathcal{N}=2$ SQCD}

We begin with a couple of warm-ups involving the four-point one-loop $\mathcal{N}=2$ SQCD amplitudes in the representation derived in ref. [46]. As the full integration of these amplitudes is straightforward, we do not dwell on it here. Instead, we concentrate on interpreting their divergent and transcendental behavior - without performing any loop integrals explicitly, to see how this behavior stems directly from the integrands. Of course, at one loop such observations are somewhat redundant given how easily the relevant integrals may be performed. However, examination of the one-loop integrands serves to illustrate the concepts discussed in previous sections, and will prepare us for the more involved two-loop amplitudes in section 4 .

\subsection{External vectors + matter}

As a first illustration of how IR behavior operates prior to integration, we consider the one-loop $\mathcal{N}=2$ SQCD amplitude with both vectors and matter on external legs. In ref. [46] this amplitude was presented in a form that makes manifest the color-kinematics duality [47-51]. Its color-dual numerators are nonzero for boxes and triangles, but only the former give rise to non-vanishing integrals: ${ }^{8}$

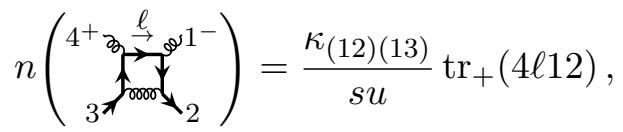

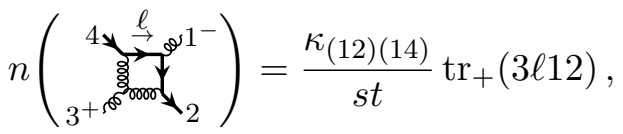

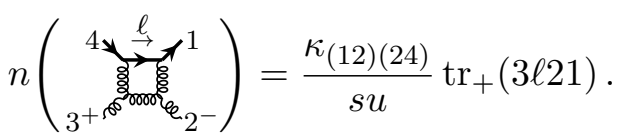

Diagrammatically, we denote gluons (vector multiplets) by curly lines and the helicities for external lines. The matter flow is shown using arrowed lines, such that the arrow direction corresponds to the chirality of a quark (matter hypermultiplet).

A remarkable property of the above numerators is that they vanish whenever the loop momentum associated with any matter edge goes to zero. Similarly, the numerators vanish

\footnotetext{
${ }^{7}$ In this paper we omit the antisymmetric echo of the six-dimensional Levi-Civita tensor $\epsilon\left(\mu_{i}, \mu_{j}\right)$ defined in refs. [45, 46], as it always integrates to zero.

${ }^{8}$ Numerators with reversed arrows are given by matter-reversal symmetries, see ref. [46] for details.
} 
if a loop momentum becomes collinear to one of the external vectors, but not the external matter. For instance, the numerator (3.1b) vanishes when $\ell \rightarrow 0$, but not when $\ell+p_{4} \rightarrow 0$; it also vanishes when $\ell \| p_{1}$, but not when $\ell \| p_{4}$. These properties will remain true for all kinematic numerators explicitly shown in this paper.

The full color-dressed amplitude is expressed in terms of these three diagrams as

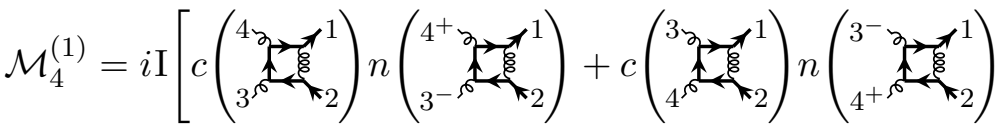

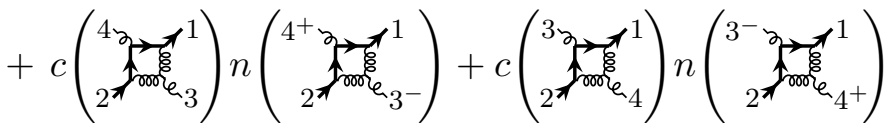

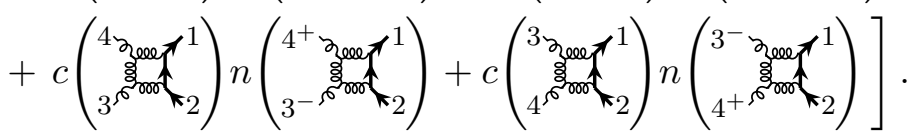

In the following we will inspect the IR divergence structure of the amplitude (3.2) and derive the IR factorization formula (2.22a). To do that, we analyze the soft and collinear regions of individual diagrams in a way that is similar to what underlies the strategy of expansion by regions [99-102], as well as the recent IR subtraction method of ref. [103].

Consider for concreteness the first diagram

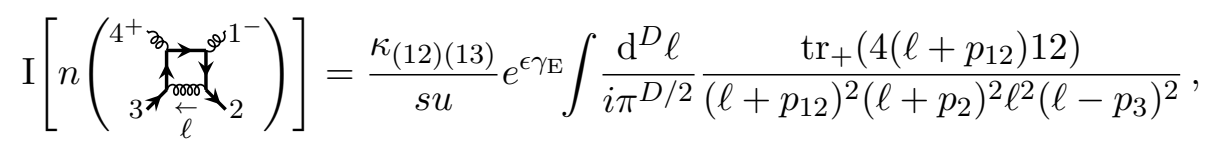

where we have relabeled the loop momentum $\ell$ for convenience. This integral diverges in three regions: one soft, $\ell \rightarrow 0$, and two collinear, $\ell \| p_{2}$ and $\ell \| p_{3}$. In all of those regions, we find that the integrand is approximated by a single function:

$$
\begin{aligned}
\frac{\operatorname{tr}_{+}\left(4\left(\ell+p_{12}\right) 12\right)}{\left(\ell+p_{12}\right)^{2}} & \stackrel{\ell \rightarrow 0}{\longrightarrow} \frac{\operatorname{tr}_{+}\left(4 p_{12} 12\right)}{s}=u, \\
& \stackrel{\ell \rightarrow \tau p_{2}}{\longrightarrow} \frac{\operatorname{tr}_{+}\left(4\left(p_{1}+(1+\tau) p_{2}\right) 12\right)}{\left(p_{1}+(1+\tau) p_{2}\right)^{2}}=u, \\
& \stackrel{\ell \rightarrow \tau p_{3}}{\longrightarrow} \frac{\operatorname{tr}_{+}\left(4\left(p_{12}+\tau p_{3}\right) 12\right)}{\left(p_{12}+\tau p_{3}\right)^{2}}=u .
\end{aligned}
$$

In all other potentially soft or collinear regions the integral vanishes due to the presence of the trace factor, e.g.

$$
\operatorname{tr}_{+}\left(4\left(\ell+p_{12}\right) 12\right) \stackrel{\ell+p_{2} \rightarrow 0}{\longrightarrow} 0
$$

Focusing on the divergent behavior, we may replace $\operatorname{tr}_{+}\left(4\left(\ell+p_{12}\right) 12\right) /\left(\ell+p_{12}\right)^{2} \rightarrow u$ at the integrand level. Ignoring finite $\mathcal{O}\left(\epsilon^{0}\right)$ terms, we retrieve a scalar triangle diagram:

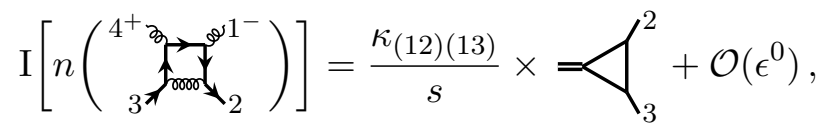


where we have used the integral notation for the triangle integral previously introduced in eq. (2.30). The other two diagrams are similarly expressed as

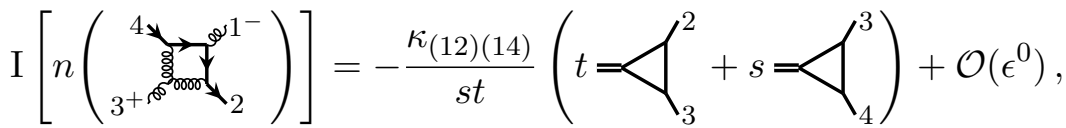

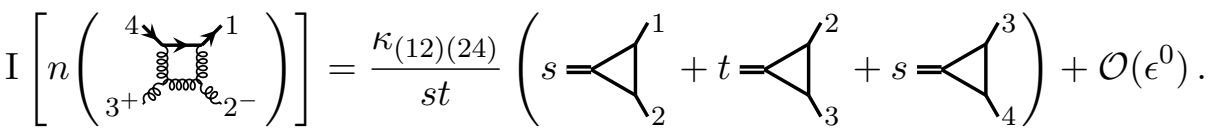

Each triangle is associated with a soft region on the left-hand side, corresponding to when gluons are exchanged on the outside edge. ${ }^{9}$

In terms of color factors the IR divergent regions are naturally represented by the dipole operator $\mathbf{T}_{i} \cdot \mathbf{T}_{j}$, which attaches a gluon bridge to the tree-level factors. We find it illuminating to use color diagrams to illustrate this point. For instance, the three diagrams in eq. (3.6) can be obtained from tree-level color factors as follows:

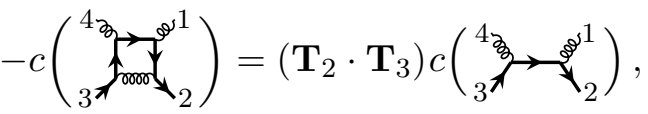

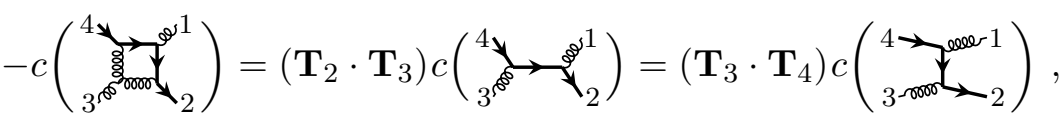

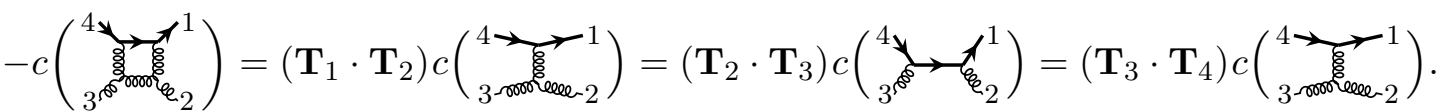

The idea here is that one should insert the appropriate relation for a particular soft region. Putting the pieces together, it is then a simple exercise to show that ${ }^{10}$

$$
\mathcal{M}_{4}^{(1)}=\left(\sum_{i<j}^{4} s_{i j}=\bigcup_{j}^{i} \mathbf{T}_{i} \cdot \mathbf{T}_{j}\right) \mathcal{M}_{4}^{(0)}+\mathcal{M}_{4}^{(1) \mathrm{fin}},
$$

where $\mathcal{M}_{4}^{(1) \text { fin }}=\mathcal{O}\left(\epsilon^{0}\right)$, and the tree-level amplitude may be written in the color basis of Del Duca, Dixon, and Maltoni [105] as

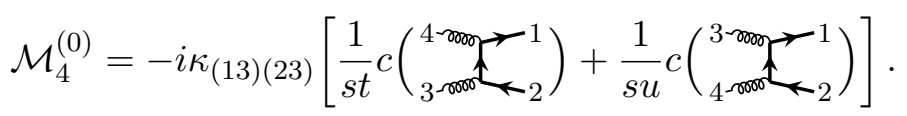

In this way, we have derived the one-loop IR factorization formula (2.22a) for the anomalous dimensions $\gamma_{g}^{(1)}=-\beta_{0} / 2$ and $\gamma_{q}^{(1)}=\gamma_{\bar{q}}^{(1)}=0$, as given by eq. (2.35). By promoting $\mathbf{S}(\epsilon)$ to include a full triangle integral we have incorporated $\mathcal{O}\left(\epsilon^{0}\right)$ terms into the finite piece, so $\mathcal{M}_{4}^{(1) \text { fin }} \neq \mathcal{H}_{4}^{(1)}$ when comparing with eq. (2.22a).

\footnotetext{
${ }^{9}$ We have implicitly made a choice in eq. (3.4) when we decided not to apply the kinematic limits to the "eikonal" propagators which in the soft limit would become linear, $\left(\ell+p_{2}\right)^{2} \rightarrow 2 \ell \cdot p_{2},\left(\ell-p_{3}\right)^{2} \rightarrow-2 \ell \cdot p_{3}$. This allowed us to avoid spurious UV divergences and at the same time to implement the collinear divergences into the same approximating function as the soft ones. This choice is consistent with the prescription of ref. [104], also recently used in ref. [103].

${ }^{10}$ One also requires the commutation relation
}

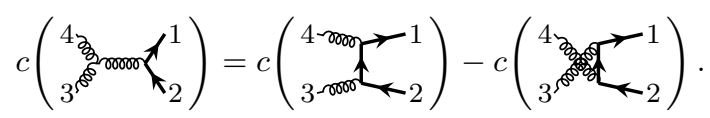


Let us now comment on the transcendentality structure of this amplitude. Performing integrand reduction on the three numerators in eq. (3.1) we see that only scalar box and triangle integrals appear. As both of these have uniform transcendental weight, $\mathcal{M}_{4}^{(1)}$, $\mathcal{M}_{4}^{(1) \text { fin }}$, and $\mathcal{H}_{4}^{(1)}$ all contain only weight-2 terms at $\mathcal{O}\left(\epsilon^{0}\right)$. So in this example the choice of IR scheme has no bearing on transcendentality. The uniform weight property is linked to the absence of scalar bubble integrals, which in this case happens without the need to specialize to the conformal theory where $N_{f}=C_{A} / T_{F}$. The dependence on number of flavors $N_{f}$, encoded by $\beta_{0}=C_{A}-N_{f} T_{F}$, cancels between $\beta_{0}$ and $2 \gamma_{g}^{(1)}$, which is reflected by the absence of closed matter loops in the integrand (3.2).

In this exercise we have seen how an analysis of soft regions can be used to expose the IR behavior of one-loop amplitudes. A similar exercise works for the one-loop four-quark amplitude; however, in that case the color-dual numerators presented in ref. [46] have soft and collinear divergences in undesirable regions associated with matter lines. Some rearrangement of the integrand is necessary, which has the unfortunate side-effect of spoiling color-kinematics duality. In the next example, involving only external gluons, we will see how relaxing the duality yields an integrand well suited for our analysis of the divergence structure.

\subsection{External vectors}

In refs. [49, 81, 106-109] the one-loop four-gluon integrand was presented in a form satisfying color-kinematics duality. By allowing ourselves to violate the duality, we write down the integrand in a form that more readily exhibits its expected IR behavior:

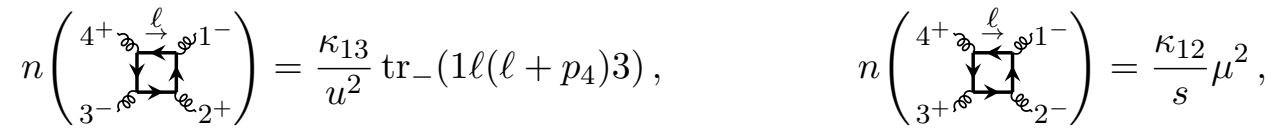

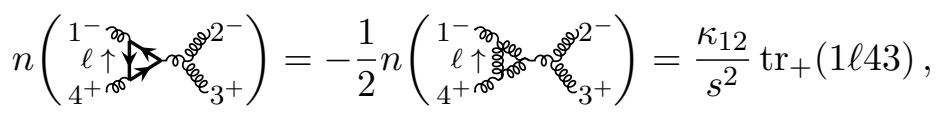

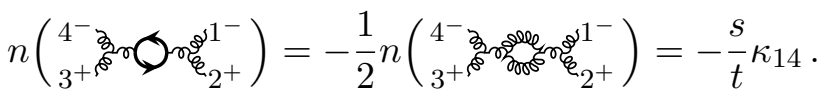

Recall that our external-state notation involving $\kappa_{i j}$ lets us add multiple helicity configurations within the same object. For example, the matter bubble numerator can be promoted to

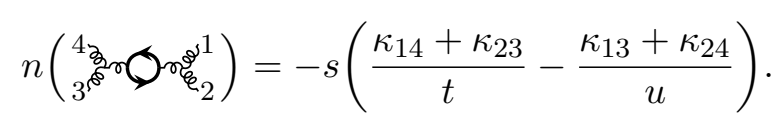

The only non-vanishing numerator absent from eq. (3.10) is the purely vector box; it is given by the $\mathcal{N}=4$ matching identity

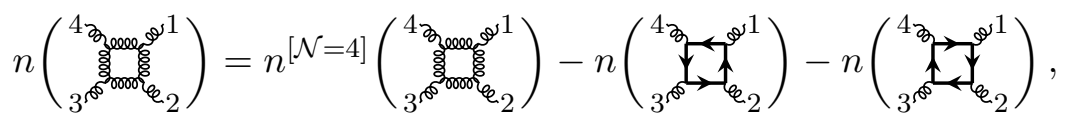

where the two matter numerators are equal. The $\mathcal{N}=4$ box numerator is explicitly

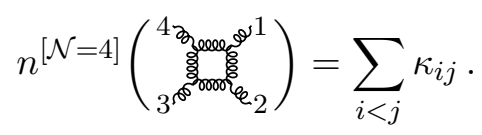


As discussed in section 2.5, identities of the type (3.12) follow from the fact that after stripping away the color information, the states of the $\mathcal{N}=2$ vector and matter multiplets add up to a single $\mathcal{N}=4$ multiplet.

An advantage of the above diagrammatic representation is that the triangle integrals vanish to all orders in $\epsilon$ due to basic symmetry arguments. Using these expressions, let us consider the divergent behavior of the amplitude:

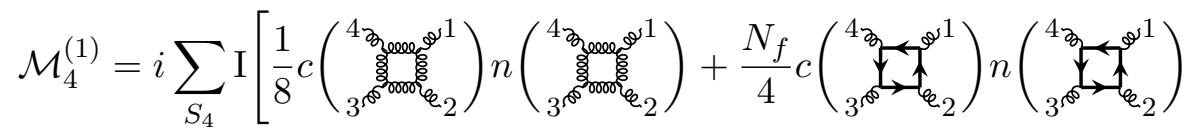

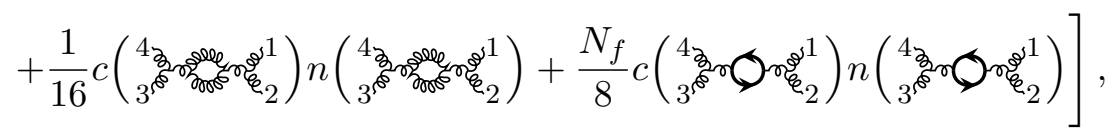

where the sum is over permutations of external legs. For comparison, the corresponding $\mathcal{N}=4$ SYM amplitude consists only of the box (3.13):

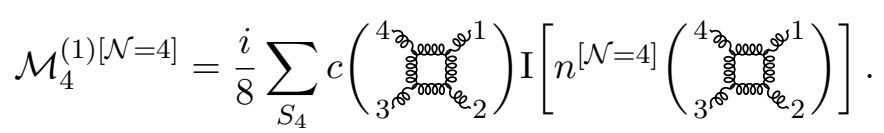

The remainder function $\mathcal{W}_{4}^{(1)}=\mathcal{M}_{4}^{(1)}-\mathcal{M}_{4}^{(1)[\mathcal{N}=4]}$ (see section 2.3) is then given as:

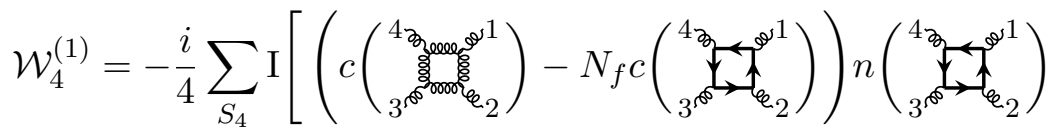

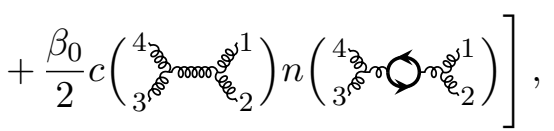

where we have reinstated $\beta_{0}=C_{A}-N_{f} T_{F}$ by factoring out the Casimir values from the bubble color factors.

In the box numerators (3.10a), the $\mu^{2}$ components integrate to $\mathcal{O}(\epsilon)$ terms. In the other helicity configurations it is the Dirac traces that block all potentially IR-singular regions without introducing any additional UV divergences, so all box contributions are finite. Therefore, $\mathcal{W}_{4}^{(1)}$ only diverges in the UV due to the bubble integrals:

$$
=\mathrm{O}=\frac{r_{\Gamma}}{\epsilon(1-2 \epsilon)}\left(-p^{2}\right)^{-\epsilon}=\frac{1}{\epsilon}+2-\log \left(-p^{2}\right)+\mathcal{O}(\epsilon) .
$$

One can now easily show that the permutation sum in eq. (3.16) leads to the correct factorization:

$$
\mathcal{W}_{4}^{(1)}=-\frac{\beta_{0}}{\epsilon} \mathcal{M}_{4}^{(0)}+\mathcal{H}_{4}^{(1)}-\mathcal{H}_{4}^{(1)[\mathcal{N}=4]},
$$

where the tree-level amplitude in this case is

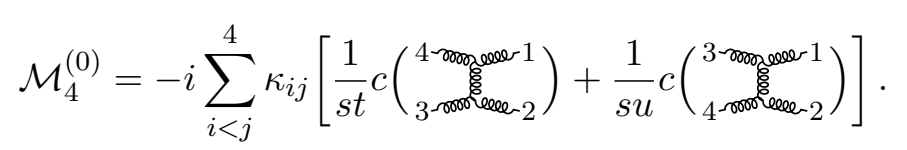

As predicted in section 2.3, the IR behavior of the one-loop four-vector amplitude is entirely captured by $\mathcal{N}=4 \mathrm{SYM}$. As for the UV, the bubble integral (3.17) contributes weight- 0,1 terms to the $\mathcal{O}\left(\epsilon^{0}\right)$ part of $\mathcal{H}_{4}^{(1)}$ which violate uniform transcendentality. 
Might there exist a better UV-subtraction scheme that ameliorates the transcendentality properties? One could be tempted to promote the $1 / \epsilon$ divergence in eq. (3.18) to a full bubble integral and thus absorb the unwanted extra terms in $\mathcal{H}_{4}^{(1)}$. However, this would introduce additional kinematic dependence of the form $\log \left(-p^{2}\right)$ into the subtraction, and whatever color-space operator acts on the tree-level amplitude must be symmetric on $s, t$, and $u$ - therein lies the problem. For instance, the only $s$-channel bubble in $\mathcal{W}_{4}^{(1)}$ has a kinematic numerator given in eq. (3.11); as it does not contain all six $\kappa_{i j}$ components, it cannot be made proportional to the tree amplitude in all external helicity configurations at once. So no sensible color-space operator, kinematically depending on bubbles and acting on $\mathcal{M}_{4}^{(0)}$, manages to reproduce the desired behavior.

We must therefore conclude that, unless we specialize to the conformal theory where $\beta_{0}=0$, the lower-weight terms introduced by bubble integrals to the one-loop amplitudes cannot be subtracted. This will also be true for less supersymmetry and more external legs. At two loops, our ability to incorporate bubbles into two-loop triangle integrals will enable us to subtract them consistently, and thus radically improve the transcendentality properties of the amplitudes.

\section{IR structure of two-loop $\mathcal{N}=2$ SQCD}

In this section we examine the IR behavior of the two-loop four-point amplitudes prior to full integration. This precedes our analysis of their transcendentality properties after integration, which we will do in section 5. In the four-gluon case, we use the compact form of the integrand presented in ref. [46], which is well-suited to the analysis of its pole structure; here we expose it at the level of individual diagrams before integration. This provides us with a strong motivation of the IR decomposition presented in eq. (2.32). For the mixed amplitude, we show how — by relaxing the constraints imposed by colorkinematics duality - the integrand can be reorganized into a form making use of similar Dirac traces, which again highlights the structure.

\subsection{External vectors}

The full two-loop four-vector amplitude may be expressed in terms of ten diagrams of four topologies, none of which vanish upon integration:

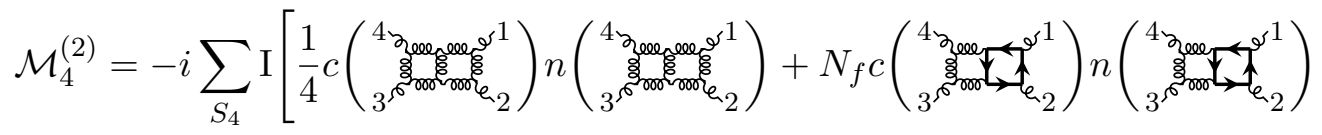

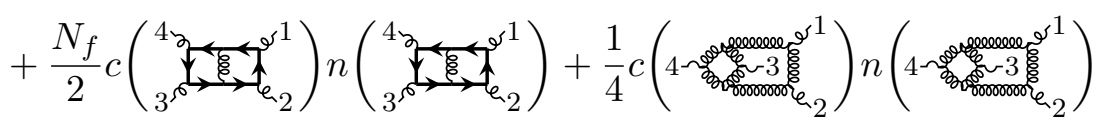

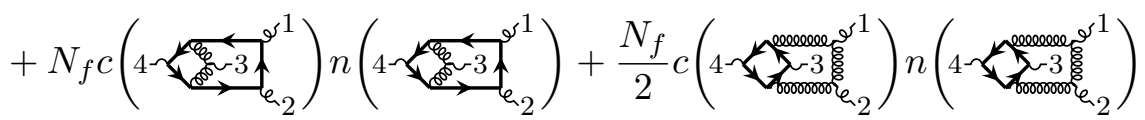

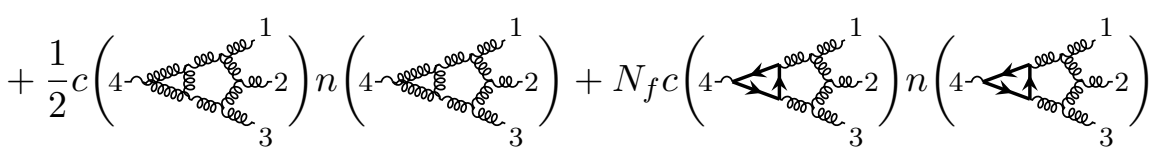

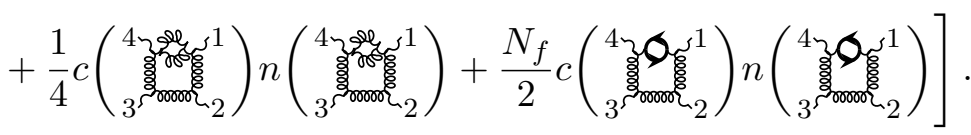


We could try to analyze this amplitude using the minimal factorization formulae $(2.22)$ or (2.24), involving poles ranging from $1 / \epsilon$ to $1 / \epsilon^{4}$. It is, however, much more appealing to use the more transparent formula (2.28), which holds after we subtract the maximally supersymmetric amplitude

$$
\begin{aligned}
& \mathcal{M}_{4}^{(2)[\mathcal{N}=4]}
\end{aligned}
$$

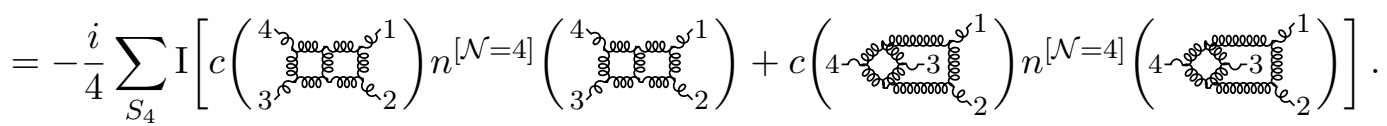

We are aided by the $\mathcal{N}=4$ matching conditions, which the kinematic numerators satisfy by construction $[45,46]$. For instance, the following combination of two-loop non-planar numerators add up to a single $\mathcal{N}=4$ numerator:

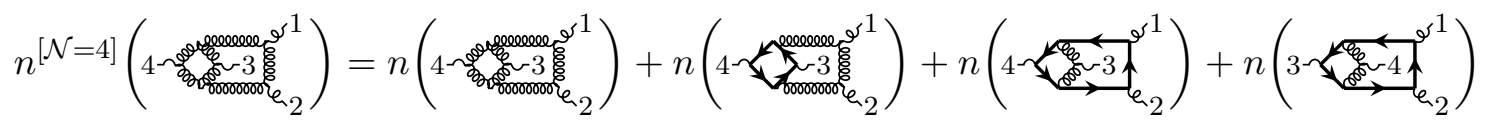

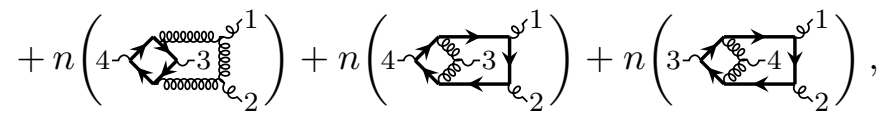

and similarly for the other three topologies (two of which are zero in $\mathcal{N}=4 \mathrm{SYM}$ ). These identities allow us to express the two-loop remainder in terms of only six kinematic numerators:

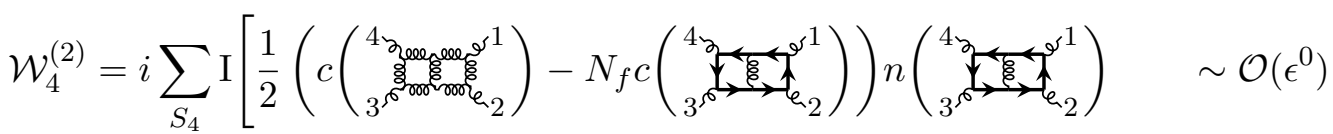

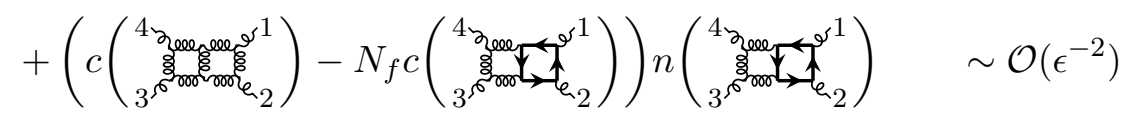

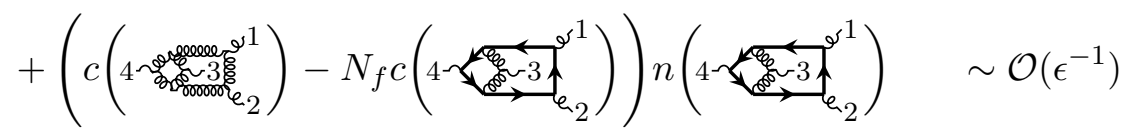

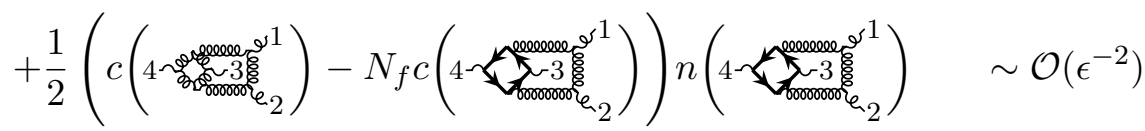

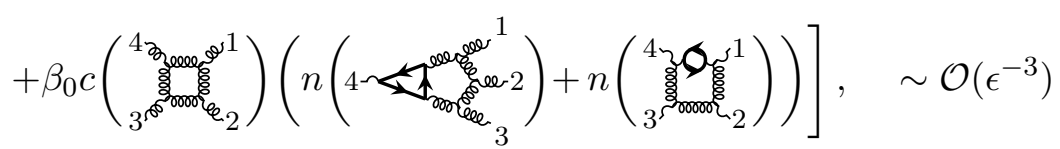

where to the right of each line we have displayed its divergent behavior. Ahead of the detailed analysis below, let us point out the prominent features of the remainder (4.4).

Due to the IR-blocking numerator properties there is no overlap of different soft, collinear, or UV regions in any of the remaining diagrams, as all of them contain a closed matter loop. Indeed, by using the $\mathcal{N}=4$ matching identities we have eliminated exactly the four diagrams that did not have any such loops and therefore incorporated the leading $1 / \epsilon^{4}$ divergences - shared with the subtracted $\mathcal{N}=4$ amplitude.

Note that the color factors for the two topologies in the last line of eq. (4.4) have been rearranged with respect to eq. (4.1). They coincide up to certain Casimir factors, which 
leads to a natural appearance of the beta-function coefficient. This is consistent with the fact that these topologies, which we dub "pentagon-triangle" and "box-bubble", are the only ones found to be UV-divergent. Their UV divergences come from the closed matter loops and enhance the $1 / \epsilon^{2}$ IR divergence behavior of the gluonic part of the diagrams to an overall $1 / \epsilon^{3}$.

The leading IR divergence rates of the other four diagrams, as indicated in eq. (4.4), can be understood from the IR-blocking numerator properties, which only allow vector propagators to produce poles in $\epsilon$.

As derived from the general principles in section 2, the remainder (4.4) must obey the factorization formula (2.32). Since the amplitude has already been fully integrated in ref. [40], for illustrative purposes we find it sufficient to perform an integrand-level proof of the factorization up to and including terms $\mathcal{O}\left(\epsilon^{-2}\right)$. At this level, we can further simplify the formula by noticing that the two-loop triangles (2.31) satisfy

$$
\mathcal{Q}_{j}^{i}=2=\oint_{j}^{i}+\mathcal{O}\left(\epsilon^{-1}\right)
$$

up to the first two orders in $\epsilon$. It is therefore sufficient to prove

$$
\mathcal{W}_{4}^{(2)}=\left(\sum_{i<j}^{4} s_{i j}=\mathcal{L}_{j}^{i} \mathbf{T}_{i} \cdot \mathbf{T}_{j}\right) \mathcal{W}_{4}^{(1)}+\beta_{0}\left(\sum_{i<j}^{4} s_{i j}=\oint_{j}^{i} \mathbf{T}_{i} \cdot \mathbf{T}_{j}\right) \mathcal{M}_{4}^{(0)}+\mathcal{O}\left(\epsilon^{-1}\right)
$$

where expressions for $\mathcal{W}_{4}^{(1)}$ and $\mathcal{M}_{4}^{(0)}$ are given in eqs. (3.16) and (3.18), respectively.

Below we explain how to interpret the divergent behavior of each of the integrals appearing in the two-loop remainder $\mathcal{W}_{4}^{(2)}$. Once the integrals have been decomposed into their various divergent regions, it is a simple (albeit cumbersome) task to assemble them and reproduce eq. (4.6).

\subsubsection{Planar and non-planar double boxes}

Up to relabelings of their loop momenta, the following three planar and non-planar doublebox numerators are equal in any helicity configuration and are given by

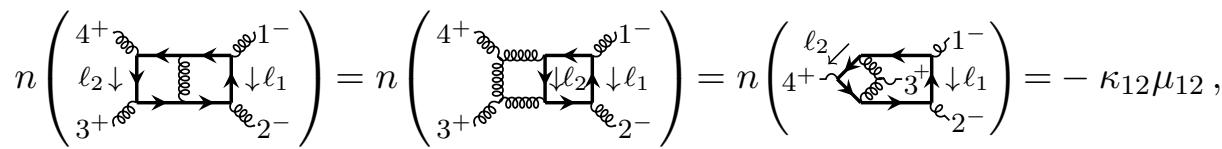

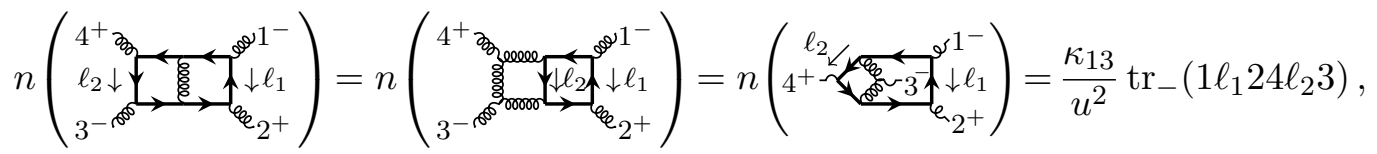

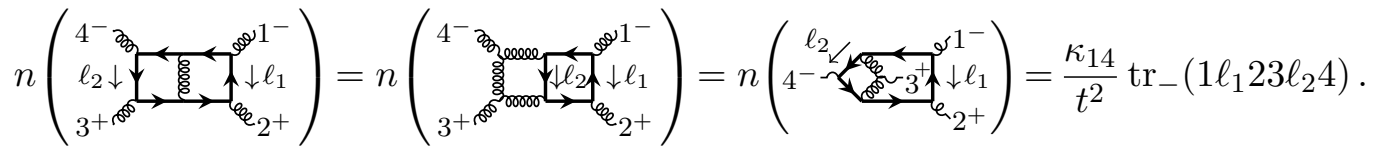

The state configurations with flipped helicities, which are not shown, can be obtained by an appropriate replacement of the $\kappa$-prefactors $\left(\kappa_{12} \rightarrow \kappa_{34}\right.$ and so on) and switching the parity of the traces $\operatorname{tr}_{-} \rightarrow \operatorname{tr}_{+}$. 
The integrals of all three numerators in the first column are entirely finite. Indeed, setting the loop momentum of any internal edge carrying hypermultiplets to zero forces the corresponding numerator to vanish; similarly for any collinear region that might otherwise have produced a divergence. The overall powers of $\ell_{1}$ and $\ell_{2}$ are also too low to produce UV divergences. Finite expressions for the integrals involving six-term traces were obtained by Caron-Huot and Larsen in ref. [110]; the integral of $\mu_{12}$ can be done using a Schwinger parametrization, as we shall explain in section 5. For the present purpose of understanding divergences, we can safely ignore these integrals, which correspond to the first line of eq. (4.4).

A similar examination of the non-planar numerators in the third column of eq. (4.7) reveals that the only permitted divergence is in the collinear region $\left(\ell_{12}+p_{1}\right) \| p_{3}$. It naturally arises from the three-gluon vertex in the center of the diagram. However, as it only gives rise to an $\mathcal{O}\left(\epsilon^{-1}\right)$ divergence we do not require its precise form for our present analysis.

As for the three diagrams in the second column of eq. (4.7), their integrals behave as $\mathcal{O}\left(\epsilon^{-2}\right)$ due to the possibility of simultaneous soft and collinear divergences in the gluonic rung on the left. An integrand analysis lets us effortlessly extract the leading order in $\epsilon$ (see also refs. [16, 52]):

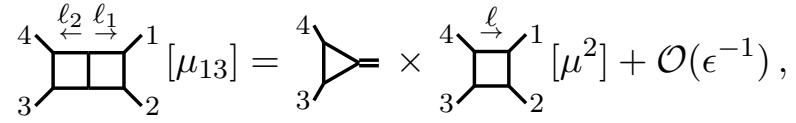

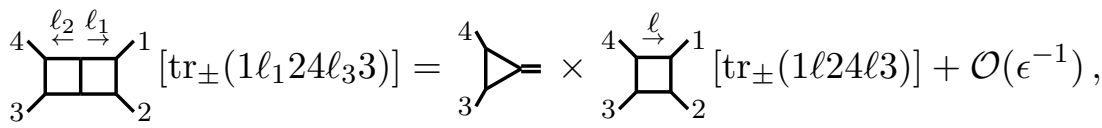

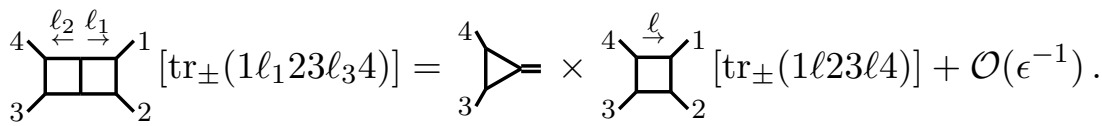

where we have relabelled the loop momenta and used $\ell_{3}=\ell_{1}+\ell_{2}$. The six-term traces that emerge as the one-loop box numerators on the right-hand side are familiar to us, as they occur in the unitarity cuts of the one-loop amplitude presented in ref. [46]. Together with the appearance of the triangle integral, this implies that we have found terms belonging to $\mathcal{W}_{4}^{(1)}$.

The other non-planar double boxes occurring in eq. (4.4) are

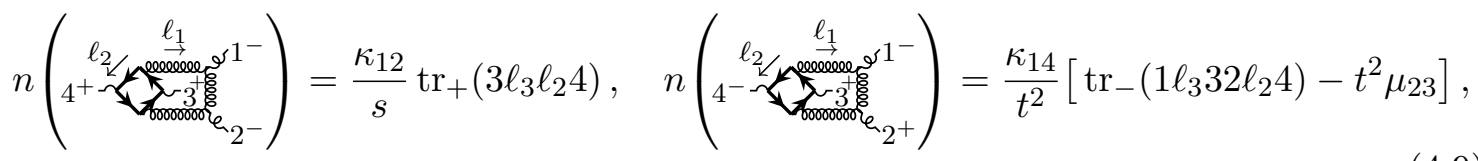

where $\ell_{3}=\ell_{1}+\ell_{2}$. These permit both soft and collinear divergences in the gluonic $\ell_{1}$ loop. As before, we can extract the leading divergent behavior at the integrand level:

$$
\begin{aligned}
& \stackrel{\ell_{2}}{\stackrel{\ell_{1}}{\rightarrow}} \underbrace{1}_{3}\left[\operatorname{tr}_{ \pm}\left(2 \ell_{3} \ell_{2} 4\right)\right]={ }_{3}^{4} \stackrel{\stackrel{\ell}{\longrightarrow}}{\longrightarrow_{2}^{1}}\left[\operatorname{tr}_{ \pm}\left(2\left(\ell-p_{1}\right) \ell 4\right)\right] \times=\mathcal{Y}_{3}^{1}+\mathcal{O}\left(\epsilon^{-1}\right),
\end{aligned}
$$

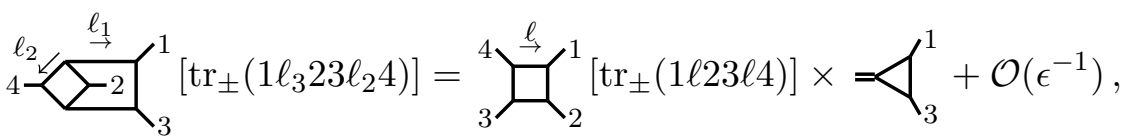

$$
\begin{aligned}
& \stackrel{\ell_{2}}{\stackrel{\ell_{1}}{\rightarrow}} \underbrace{1}_{3}\left[\mu_{23}\right]={ }_{3}^{4} \stackrel{\ell}{\rightarrow})_{2}^{1}\left[\mu^{2}\right] \times=\nvdash_{3}^{1}+\mathcal{O}\left(\epsilon^{-1}\right) \text {. }
\end{aligned}
$$


In the first case we observe that $\operatorname{tr}_{ \pm}\left(2\left(\ell-p_{1}\right) \ell 4\right)=\operatorname{tr}_{\mp}\left(1 \ell\left(\ell+p_{4}\right) 3\right)$ is precisely the factor appearing in the one-loop box diagram (3.10a). All terms on the right-hand side are natural when assembling $\mathcal{W}_{4}^{(1)}$, and again the triangles belong to the dipole operator.

\subsubsection{UV-divergent topologies}

We continue the analysis with numerators that carry the UV divergence of the remainder in eq. (4.4), namely the pentagon-triangle and the box-bubble diagrams. The numerator of the box-bubble diagram is simply

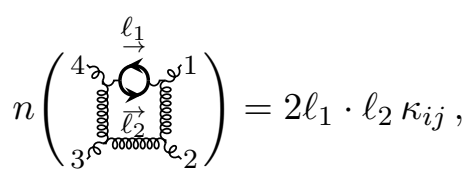

where $i, j$ are the legs carrying negative helicity. This numerator is entirely reducible, since it can be rewritten as $2 \ell_{1} \cdot \ell_{2}=\ell_{3}^{2}-\ell_{1}^{2}-\ell_{2}^{2}$ in terms of three propagator denominators. Two of the three resulting scalar integrals contain scaleless tadpoles and therefore vanish. The remaining scalar integral has four soft regions around the limits $\ell_{3} \rightarrow 0, \ell_{3} \rightarrow p_{1}$, $\ell_{3} \rightarrow p_{12}$, and $\ell_{3} \rightarrow-p_{4}$. Taking these limits at the integrand level, we extract the finite denominators from the integrals and obtain

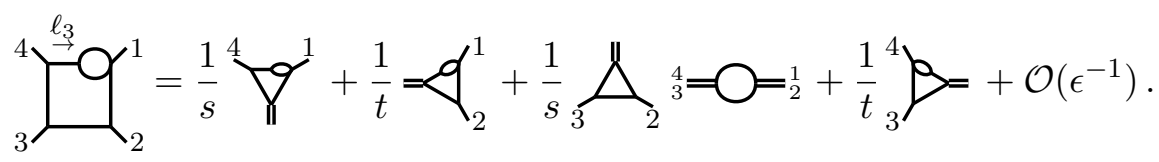

The UV-divergent internal bubble cleanly decouples from the outer integral only in the third region $\ell_{3} \rightarrow p_{12}$. The other regions naturally produce the two-loop triangle topologies that appear in the IR factorization formula (2.32). We have confirmed that the expansion (4.12) holds for two orders in $\epsilon$ by numeric evaluation in FIESTA [111-113]. Recall that the $\kappa$ prefactor contains an ordered tree amplitude and we derive

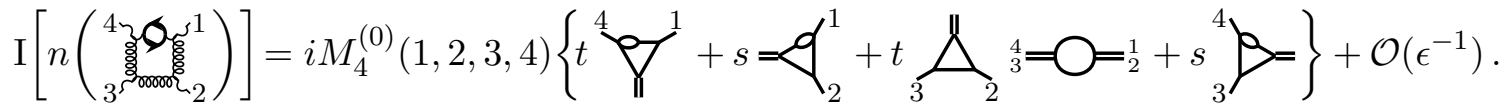

This equation is true for all gluonic helicity configurations and can be made to explicitly incorporate them all using the symbolic amplitude expression $i M_{4}^{(0)}=\frac{1}{s t} \sum_{i<j} \kappa_{i j}$.

The pentagon-triangle contributions to eq. (4.4) have numerators of the form

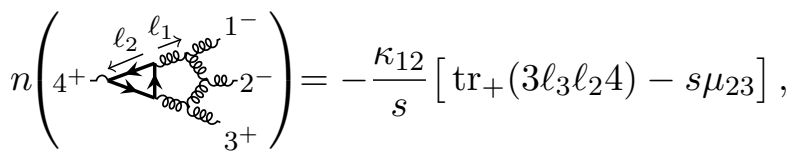

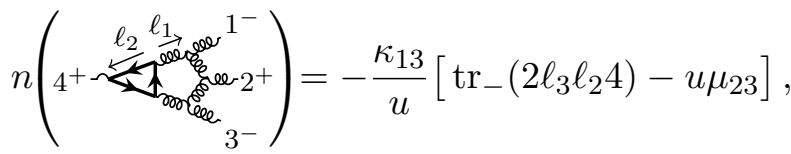

where again $\ell_{3}=\ell_{1}+\ell_{2}$. Since the chirally projected Dirac traces are taken strictly in the four-dimensional sense, the $\mu$-terms can be regarded as their extra-dimensional components. 
Although soft and collinear divergences in the $\ell_{2}$ loop are blocked by the numerators, UV poles do develop in the $\ell_{2} \rightarrow \infty$ limit. Taken in combination with simultaneous soft and collinear singularities in the $\ell_{1}$ loop, this implies that leading poles occur at $\mathcal{O}\left(\epsilon^{-3}\right)$.

Considering the soft limits of a slightly more general integral, we find

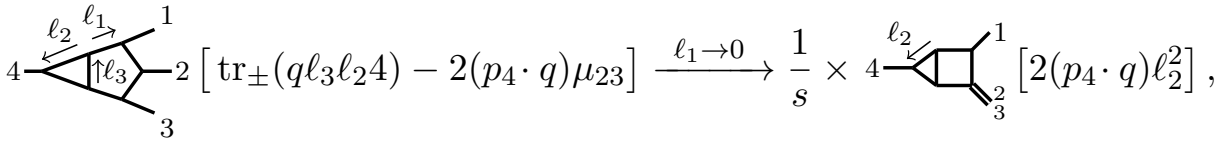

$$
\begin{aligned}
& \stackrel{\ell_{1} \rightarrow p_{1}}{\longrightarrow} \frac{1}{t} \times \stackrel{\ell_{2}}{\ell_{1}}\left[2\left(p_{4} \cdot q\right) \ell_{2}^{2}\right] \times=\mathbf{Y}_{2}^{1}
\end{aligned}
$$

For $\ell_{1} \rightarrow 0$ we factor out $\left(\ell_{1}-p_{12}\right)^{2} \rightarrow s$, while the four-dimensional trace and $\mu_{23} \rightarrow \mu_{22}$ combine into a $D$-dimensional $\ell_{2}^{2}$ in the numerator. In the limit $\ell_{1} \rightarrow p_{1}$ the propagators factorize into two one-loop topologies, and we drop the numerator contribution $\operatorname{tr}_{ \pm}\left(q 1 \ell_{2} 4\right)$ that integrates to zero. Analogous simplifications happen for the regions $\ell_{1} \rightarrow p_{12}$ and $\ell_{1} \rightarrow-p_{4}$. By integrand reduction we obtain

$$
\begin{aligned}
& \underbrace{\stackrel{\ell_{2}}{\ell_{1}} \overbrace{1}^{t} \ell_{3}}_{3}\left[\operatorname{tr}_{ \pm}\left(q \ell_{3} \ell_{2} 4\right)-2\left(p_{4} \cdot q\right) \mu_{23}\right]
\end{aligned}
$$

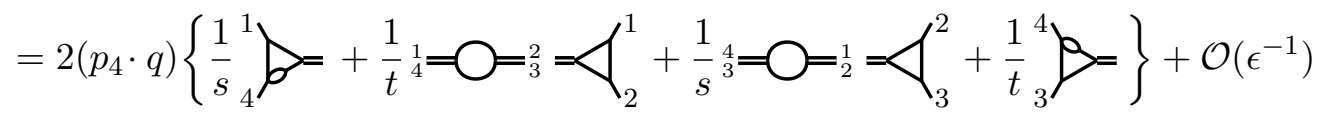

involving the two-loop triangles (2.31b). Again, we have checked this expansion numerically using FIESTA [111-113].

That our analysis of soft regions correctly predicts both the $1 / \epsilon^{3}$ and $1 / \epsilon^{2}$ terms in eqs. (4.12) and (4.16) is non-trivial. Unlike section 4.1.1, where the leading divergent behavior $\mathcal{O}\left(\epsilon^{-2}\right)$ was always associated with a particular soft region, here one can imagine $1 / \epsilon^{2}$ poles arising from a collinear divergence in the $\ell_{1}$-loop and a UV divergence in the $\ell_{2}$-loop. Such terms would not be detected merely by thinking about soft regions, yet the above analysis leaves nothing out. It seems to be a characteristic of these trace-based numerators that they permit such behavior. For the pentagon-triangle numerators (4.14), the integral expansion (4.16) implies

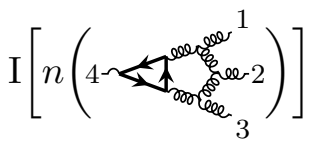

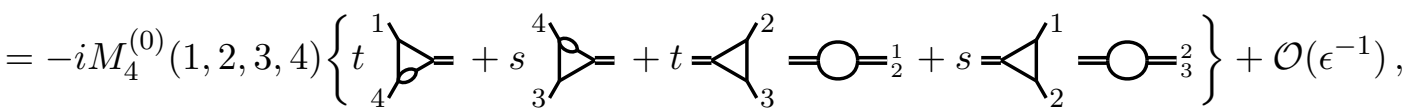

in terms of the ordered tree-level amplitude with arbitrary external helicities.

This provides us with the last ingredient needed to prove the IR factorization formula (4.6). At this order in $\epsilon$, the second two-loop triangle topology (2.31b) cleanly cancels when the pentagon-triangle and box-bubble contributions are combined. Adding them to the leading-order divergences of the planar and non-planar double boxes we find perfect agreement with eq. (4.6). 


\subsection{External vectors + matter}

In the two-loop four-gluon amplitude we see a clear link between the choice of IR subtraction scheme and the structure of the integrand. Therefore, we naturally question whether a similarly revealing choice of integrand basis might shed light on what IR-subtraction scheme best enhances the transcendentality properties of amplitudes with external matter. This question was already explored in ref. [46] to a certain extent; however, the requirement that all numerators be color-dual limited our freedom to explore other possibilities. As we have seen in previous examples, such as the one-loop four-gluon amplitude in section 3.2, relaxing color-kinematics duality can be beneficial for exposing the divergent structure of these amplitudes before integration. So here we briefly investigate this possibility.

The following two double boxes were presented in ref. [46]:

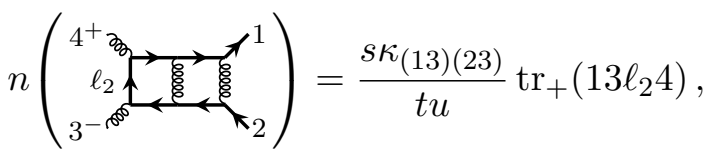

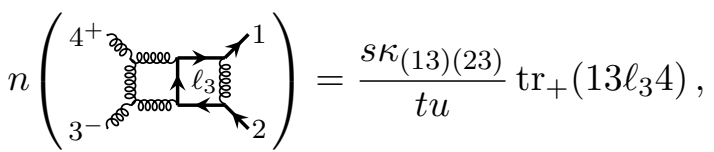

where loop momenta follow the directions of the matter arrows. These belong to the color-dual presentation of the four-point mixed amplitude (which in section 5 we will fully integrate). Allowing ourselves to relax BCJ duality, we find similarly compact expressions for the other six double boxes:

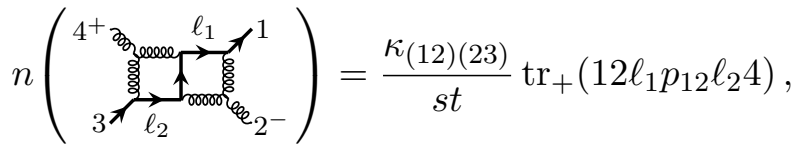

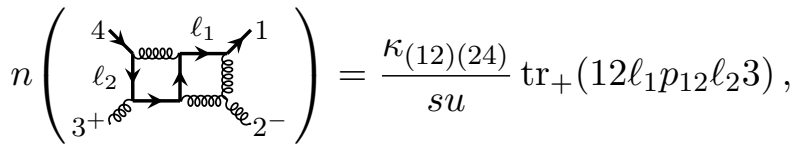

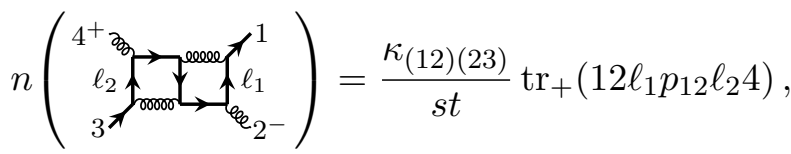

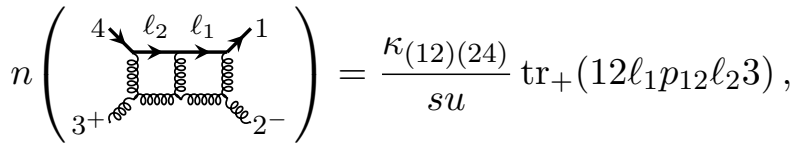

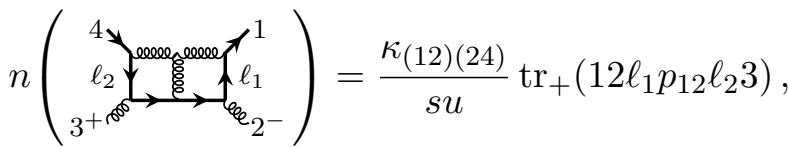

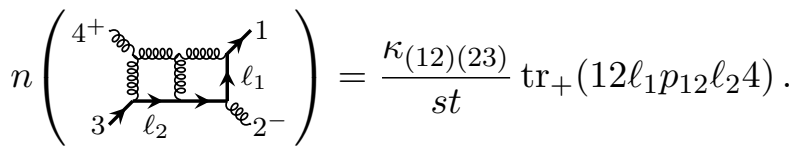

These numerators vanish whenever the loop momentum carried by an internal matter edge vanishes. Similar tricks work for the other planar numerators, which suggests that a privileged integrand basis does exist. 
Unfortunately, while relaxing the requirement of color-kinematics duality gives us more freedom to write down desirable expressions in the planar sector, it also makes it much harder to find suitable non-planars. This is because, when enforcing the duality, nonplanar numerators are given to us automatically by Jacobi or commutation relations in terms of the planars - writing down non-planar generalized unitarity cuts is therefore unnecessary. While the iterated cut construction can be used to obtain non-planar cuts, a generic mechanism for lifting them off-shell is still lacking. This does not rule out the existence of such non-planar expressions in any way- it simply complicates the task of finding them.

Another problem is that there is no natural analogue of $\mathcal{M}_{4}^{(2)[\mathcal{N}=4]}$ for us to subtract, so the IR divergences of all graphs must be analyzed. As we shall see in section 5 , this affects our ability to write down a suitable IR subtraction scheme.

\section{Integration \& transcendental weight}

Having explored how the integrand structure reflects the IR behavior of various different amplitudes, we now proceed to study the analytic form of the three two-loop $\mathcal{N}=2$ SQCD amplitudes under consideration by direct integration. The four-vector amplitude was already integrated in ref. [40], so here we apply the same techniques to the other two amplitudes. The algorithm is as follows:

(1) All contributions are converted to scalar-type integrals using Schwinger parametrization $[114,115]$. Amongst the resulting terms are integrals in $(D+2 n)$ dimensions (where $n \in \mathbb{Z}$ ) and with raised powers of propagators.

(2) Higher-dimensional scalar integrals are shifted back to $D$ dimensions using dimensional recurrence relations $[116,117]$.

(3) Using the Mathematica package LiteRed [118], the resulting D-dimensional scalar integrals are reduced to a basis of masters using integration-by-parts relations (IBPs) [119, 120].

(4) Known expressions for the four-point two-loop master integrals [121-125] are inserted. Final manipulation of the results is performed using the Mathematica package HPL [126].

The resulting $\epsilon$-pole structure in all three cases matches the one predicted in eq. (2.22b).

We provide machine-readable text files for the complete expressions of the one- and two-loop four-point amplitudes as supplementary material attached to this paper. The expressions are written out for an $\mathrm{SU}\left(N_{c}\right)$ gauge group,${ }^{11}$ distinct flavors of external matter parton pairs, and $N_{f}$ flavors for internal matter loops. The equal-flavor amplitudes can be obtained by an appropriate summation over permutations of the distinct-flavor expressions. For the four-gluon amplitude, we present all helicity configurations as in ref. [40]. For the amplitudes involving external matter we make a particular choice w.r.t. the helicities of

\footnotetext{
${ }^{11}$ The gluonic amplitude for a selection of different gauge groups can be found attached to ref. [40].
} 
the external particles, which can be readily observed from their $\kappa$-dependence as shown in (1.8). The two-loop cusp and field anomalous dimensions have already been advertised in eq. (2.35).

Since we have the full analytic expressions at our disposal, we are able to study the range of transcendental weights appearing in the amplitudes. In ref. [40], where the analytic form of the four-gluon amplitude was studied, a conspiracy between the IR subtraction scheme as defined by Catani [15] (shown in eq. (2.15)) and terms of lower transcendentality was observed at the conformal point of the theory. More specifically, writing the gluonic remainder as [40]

$$
\mathcal{W}_{n}^{(L)}=\mathcal{R}_{n}^{(L)}+\left(C_{A}-T_{F} N_{f}\right) \mathcal{S}_{n}^{(L)},
$$

one sees $\mathcal{R}_{4}^{(2)}$ contains terms of weight 2 through 4 at $\mathcal{O}\left(\epsilon^{0}\right)$. Upon IR subtraction via

$$
\mathcal{R}_{4}^{(2)}=\left(\sum_{i<j}^{4} s_{i j}=<_{j}^{i} \mathbf{T}_{i} \cdot \mathbf{T}_{j}\right) \mathcal{R}_{4}^{(1)}+\mathcal{R}_{4}^{(2) \mathrm{fin}}
$$

it was found that $\mathcal{R}_{4}^{(2) \text { fin }}$ is given by terms of weights 3 and 4 only at $\mathcal{O}\left(\epsilon^{0}\right)$. This intriguing cancellation was limited only to $\mathcal{R}_{4}^{(2)}$, as the Catani-style subtraction did not ameliorate the transcendental structure of the non-conformal part of the amplitude.

Bypassing this restriction, from the point of view of transcendentality the scheme defined in eq. (2.32) seems to be a logical extension of eq. (5.2). Indeed, performing this subtraction we see that, although $\mathcal{W}_{4}^{(2)}$ contains terms of all possible weights 0 through 4 at $\mathcal{O}\left(\epsilon^{0}\right), \mathcal{W}_{4}^{(2) \text { fin }}$ is described entirely by terms of weights 3 and 4 at the same order in $\epsilon$. As an illustration, we provide explicit expressions for two independent helicity configurations of $\mathcal{W}_{4}^{(2) \text { fin }}$ in appendix B. Note that the precise form of eq. (2.32) is important in this regard. For example, if we rewrite the non-conformal term using only one two-loop triangle topology, as in eq. (4.5), this will reintroduce terms of weight 2 at $\mathcal{O}\left(\epsilon^{0}\right)$.

Our discussion of the one-loop amplitudes in section 3 provides us with some intuition for why this cancellation happens. In the one-loop mixed amplitude (discussed in section 3.1) no bubbles appeared at any stage, and the result had uniform weight regardless of our scheme choice, as this property manifestly holds for the other topologies involved. In the one-loop four-vector amplitude (discussed in section 3.2) the appearance of lowerweight terms in $\mathcal{H}_{4}^{(1)}$ was attributed to our inability to consistently remove bubble integrals. In the two-loop gluonic amplitude, while bubble integrals do appear and produce UV divergences, they occur either as sub-integrals, and so can be incorporated into the two-loop triangle topologies used to subtract IR divergences, or they naturally arrange themselves into the one-loop remainder $\mathcal{W}_{4}^{(1)}$. While this does not constitute a full integrand-level understanding of the weight grading of the two-loop result, it does motivate why one could expect the scheme in eq. (2.32) to improve the transcendental structure of the finite part of the amplitude.

Having identified this peculiar scheme for the four-gluon case, it is natural to wonder whether such an enhanced cancellation of lower-weight terms can also be achieved for the two-loop amplitudes involving external matter fields. In these cases, there is no analogous 


\begin{tabular}{|c|c|c|c|}
\hline Weights at $\mathcal{O}\left(\epsilon^{0}\right)$ & Vectors & Mixed & Matter \\
\hline $\mathcal{H}_{4}^{(2)}$ & $0,1,2,3,4$ & $0,1,2,3,4$ & $0,1,2,3,4$ \\
$\mathcal{M}_{4}^{(2) \mathrm{fin}}$ & 3,4 & $2,3,4$ & $0,1,2,3,4$ \\
$\mathcal{H}_{4}^{(2) \operatorname{conf}}$ & $2,3,4$ & 3,4 & 3,4 \\
$\mathcal{M}_{4}^{(2) \text { fin,conf }}$ & 3,4 & 3,4 & 3,4 \\
\hline
\end{tabular}

Table 2. The transcendental weights appearing for various encodings of the finite part of the two-loop amplitudes under consideration. The first row shows the weights for the hard part of the various unrenormalized two-loop amplitudes as defined by minimal subtraction (2.22b); the second row shows the weights appearing after IR subtraction as defined by eq. (5.3). The third and fourth rows show the weights of the same terms evaluated at the conformal point $N_{f}=C_{A} / T_{F}$.

result in $\mathcal{N}=4 \mathrm{SYM}$ which can be subtracted and a remainder as given by eq. (2.27) cannot be defined. To this end, we can generalize the scheme defined in eq. (2.32) as follows:

$$
\begin{aligned}
\mathcal{M}_{n}^{(2)}= & {\left[\frac{1}{2} \mathbf{S}(\epsilon) \mathbf{S}(\epsilon)+\frac{1}{2}\left(\gamma_{K}^{(2)}-4 \beta_{0}\right) \mathbf{S}(2 \epsilon)+\frac{1}{\epsilon} \sum_{i=1}^{n} \gamma_{i}^{(2)}\right] \mathcal{M}_{n}^{(0)}+\left(\sum_{i<j}^{n} s_{i j}=j_{j}^{i} \mathbf{T}_{i} \cdot \mathbf{T}_{j}\right) \mathcal{H}_{n}^{(1)} } \\
& +\beta_{0}\left(\sum_{i<j}^{n} s_{i j}\left[=\boldsymbol{g}_{j}^{i}-\oint_{j}^{i}-\frac{1}{\epsilon}=\mathcal{j}_{j}^{i}\right] \mathbf{T}_{i} \cdot \mathbf{T}_{j}\right) \mathcal{M}_{n}^{(0)} \\
& +\frac{1}{2 \epsilon}\left[\frac{n}{2} \beta_{0}+\sum_{i=1}^{n} \gamma_{i}^{(1)}\right]\left(\mathcal{M}_{n}^{(1)}+\mathbf{S}(\epsilon) \mathcal{M}_{n}^{(0)}+\mathcal{H}_{n}^{(1)}\right) \\
& +\frac{1}{\epsilon}\left[\frac{n-2}{2} \beta_{1}+\frac{\beta_{0}}{2}\left(1-\frac{\zeta_{2}}{4}\right) \sum_{i=1}^{n} \mathbf{T}_{i}^{2}\right] \mathcal{M}_{n}^{(0)}+\mathcal{M}_{n}^{(2) \mathrm{fin}},
\end{aligned}
$$

where $\mathcal{H}_{n}^{(1)}$ was defined in eq. (2.22a). Using this generalized scheme involving scalar triangle integrals, we study the transcendentality of $\mathcal{M}_{n}^{(L) \text { fin }}$ for the various amplitudes computed in this paper. Our findings are summarized in table 2.

In the four-gluon case, eq. (5.3) is similar to eq. (2.32) so the same cancellations occur. For the amplitude involving both external vector and matter fields, the scheme defined above also ameliorates the transcendental structure of the finite part. We note that in this case, the uniform transcendentality of the one-loop result simplifies the weight grading of eq. (5.3). Finally, for the amplitude with all external matter fields no cancellation is observed. However, we should not rule out the possibility of a further generalization of eq. (5.3) that could induce analogous behavior. In particular, at weights 0 and 1 the color structures and transcendental objects which appear are sufficiently restricted that the proportionality to the tree-level amplitude is remarkably simple.

\section{Summary and outlook}

In this paper we have explored a link between the degree of uniform transcendentality violation and IR-subtraction schemes in $\mathcal{N}=2$ SQCD. Taking two-loop gluonic amplitudes 
as "corrections" to uniformly transcendental $\mathcal{N}=4 \mathrm{SYM}$ amplitudes, with $\mathcal{W}_{n}^{(L)} \equiv \mathcal{M}_{n}^{(L)}$ $\mathcal{M}_{n}^{(L)[\mathcal{N}=4]}$, we find that all two-loop divergences (including UV) may be expressed in terms of scalar triangle integrals:

$$
\mathcal{W}_{n}^{(2)}=\left(\sum_{i<j}^{n} s_{i j}=<_{j}^{i} \mathbf{T}_{i} \cdot \mathbf{T}_{j}\right) \mathcal{W}_{n}^{(1)}+\beta_{0}\left(\sum_{i<j}^{n} s_{i j}\left[\mathcal{Q}_{j}^{i}-\phi_{j}^{i}\right] \mathbf{T}_{i} \cdot \mathbf{T}_{j}\right) \mathcal{M}_{n}^{(0)}+\mathcal{W}_{n}^{(2) \mathrm{fin}}
$$

The use of these integrals defines a specific IR-subtraction scheme to all orders in $\epsilon$ and cancels all transcendental weight-0,1,2 terms from $\mathcal{W}_{4}^{(2) \text { fin }}$. A similar scheme was used in ref. [40] to demonstrate the cancellation of lower-weight terms from the finite part of the same amplitude at the conformal point $\beta_{0}=2 N_{c}-N_{f}=0-$ here we have generalized the result to the full SQCD theory with arbitrary $N_{c}$ and $N_{f}$.

Our understanding of this scheme came from two opposite perspectives. Firstly, in section 2 we provided a derivation of generic formulae (2.13) and (2.22) for the two-loop IR divergences - after and before UV renormalization, respectively. These formulae are valid for any massless gauge theory in four dimensions and improve on Catani's wellknown formulae (2.15) by excluding unnecessary color structures of the form $\tilde{f}^{a b c} \mathbf{T}_{i}^{a} \mathbf{T}_{j}^{b} \mathbf{T}_{k}^{c}$. In particular, the formula (2.22) for the divergences of unrenormalized (bare) amplitudes $\mathcal{M}_{n}^{(2)}$ places UV, soft, and collinear poles on an equal footing. Even more cleanup happens for gluonic amplitudes (2.28): $\mathcal{W}_{n}^{(L)}=\mathcal{M}_{n}^{(L)}-\mathcal{M}_{n}^{(L)[\mathcal{N}=4]}$ diverges only as $1 / \epsilon^{2 L-1}$ since the leading $1 / \epsilon^{2 L}$ poles are absorbed into the uniformly transcendental $\mathcal{N}=4 \mathrm{SYM}$ amplitudes. Finally, the IR scheme (6.1) was obtained by specializing to $\mathcal{N}=2$ SQCD using our results (2.35) for the two-loop anomalous dimensions.

A more intuitive picture came from studying the IR behavior of the loop integrands. In ref. [46] an iterated cut construction was used to write down expressions for all two-loop four-point $\mathcal{N}=2 \mathrm{SQCD}$ integrands. Diagrams with internal matter lines were found to have a controlled IR behavior, so that singular regions arising from massless $i / p^{2}$ propagators are "blocked" by the appearance of local numerators that vanish in those regions. This matches our physical intuition in QCD: quarks obey Fermi-Dirac statistics, so they should be distinguishable even in the high-energy (massless) limit — soft or collinear divergences should arise only from virtual gluon exchange. The usual quark propagator $i \not p / p^{2}$ also ensures this property, for instance, as $\not p$ vanishes in the soft limit $p^{\mu} \rightarrow 0$. Supersymmetric quarks and gluons follow the same qualitative behavior.

The controlled IR behavior made it possible to analyze both the divergences and transcendentality structure of multi-loop $\mathcal{N}=2$ SQCD amplitudes before integration. In the one-loop examples discussed in section 3, this involved expressing soft and collinear divergences in terms of unintegrated triangle integrals and UV divergences in terms of bubbles. The latter, we observed, source the unwanted lower-weight terms in the finite parts of the amplitudes. For the one- and two-loop gluonic amplitudes discussed in sections 3.2 and 4.1, respectively, we used an off-shell supersymmetry decomposition to eliminate all purely-gluonic diagrams from the remainder functions $\mathcal{W}_{4}^{(L)}$; the remaining diagrams all contain internal matter loops. Analysis of the two-loop amplitude in section 4.1 gave rise 
to the two-loop triangles with UV-divergent bubbles embedded, which fit naturally into the IR scheme presented in eq. (6.1).

Finally, in this paper we also integrated the two-loop four-point $\mathcal{N}=2$ SQCD amplitudes with matter on external legs, first presented in ref. [46]. Using a generalized scheme (5.3) that allows for external matter, we found a cancellation of weight- 0,1 terms from the four-point mixed amplitude but not the four-matter amplitude - results are presented in table 2. Integration of these amplitudes also provided us with the quark anomalous dimension $\gamma_{q}^{(2)}$, as well as a cross-check of the cusp and gluonic anomalous dimensions $\gamma_{K}^{(2)}$ and $\gamma_{g}^{(2)}$, all given in eq. (2.35). All three include an arbitrary dependence on the number of matter flavors $N_{f}$. They are valid in the FDH regularization scheme [67, 68], and may in principle be converted to the 't Hooft-Veltman scheme [64] or the conventional dimensional regularization scheme [65] using the dictionary of ref. [58].

Let us now expand upon the features and consequences of our work.

Anomalous dimensions. Given our $\mathcal{N}=2$ SQCD results (2.35) for the two-loop anomalous dimensions, one could wonder whether they contain hints of their lower-supersymmetry counterparts. Indeed, the result (2.35a) for the two-loop cusp anomalous dimension is in perfect agreement with the general FDH formula

$$
\gamma_{K}^{(2)}=\left(\frac{64}{9}-2 \zeta_{2}\right) C_{A}-\frac{10}{9} T_{f} n_{f}-\frac{4}{9} T_{s} n_{s}
$$

for a gauge theory minimally coupled to $n_{f}$ Weyl fermions and $n_{s}$ real scalars. It is well known to practitioners, as it can be inferred from interpolating between the cases of QCD $\left(n_{s}=0, n_{f}=2 N_{f}, T_{f}=T_{F}[61]\right)$ and $\mathcal{N}=4 \mathrm{SYM}\left(n_{f}=4, n_{s}=6, T_{f}=T_{s}=C_{A}[60]\right)$ based on its linearity with respect to the particle content. It is difficult to write similarly generic formulae for the two-loop gluonic and quark collinear anomalous dimensions, as they are sensitive to how the adjoint scalars couple to the fundamental fermions and scalars. A supersymmetry interpolation is, however, possible for pure SYM theories, obtained by setting $N_{f}=0$. We find perfect consistency with the $0<\mathcal{N} \leq 4$ supersymmetric results of refs. $[127,128]$ for the Catani terms which survive in the leading-color limit $N_{c} \rightarrow \infty$ :

$$
K_{\mathrm{FDH}}^{[\mathrm{SYM}]}=C_{A}\left[-\zeta_{2}+(4-\mathcal{N})\right], \quad H_{g, \mathrm{FDH}}^{[\mathrm{SYM}]}=\frac{1}{4} C_{A}^{2}\left[2 \zeta_{3}+\frac{4-\mathcal{N}}{2} \zeta_{2}\right],
$$

where we have adjusted the overall prefactors to match our conventions in eq. (2.17). For arbitrary $\mathcal{N}$, we can combine eqs. (2.17) and (6.3) and easily find

$$
\gamma_{K}^{(2)}=2 C_{A}\left[-\zeta_{2}+(4-\mathcal{N})\right], \quad \gamma_{g}^{(2)}=\frac{1}{16} C_{A}^{2}\left[2 \zeta_{3}+(4-\mathcal{N}) \zeta_{2}-2(4-\mathcal{N})^{2}\right]
$$

having also used $\beta_{0}=\frac{1}{2} C_{A}(4-\mathcal{N})$. Uniform transcendentality is manifest for $\mathcal{N}=4$.

Local IR subtraction. Our analysis of the IR divergences before integration was facilitated by the controlled IR behavior of the $\mathcal{N}=2$ integrands, in which the diagram numerators naturally "blocked" IR divergences associated with certain edges. These IR-blocking properties can be regarded as a kind of local IR subtraction at the amplitude-integrand 
level, which has been a subject of significant interest in QCD [103, 104, 129]. In our approach, such a (partial) subtraction is made possible by tailoring the diagram numerators to generalized unitarity cuts exactly, which is impossible without certain Levi-Civita terms. Although such terms vanish upon integration, they often participate in loop-dependent chiral Dirac traces $\operatorname{tr}_{ \pm}(\cdots)$, which we found to be natural building blocks for well-behaved loop integrands. It would be extremely interesting to see if such guidance from unitarity cuts can also help achieving local IR subtraction in QCD.

Transcendentality. Our $\mathcal{N}=2$ results reveal an intriguing interplay between IR physics and transcendentality, which is well-studied in $\mathcal{N}=4 \mathrm{SYM}$. The conjectured uniform transcendentality property $[38,39]$ is naturally implied for integrands that can be written as so-called $d \log$ forms, which is a consequence of those integrands having only unit leading singularities (see ref. [130] for a review). Using loop-level recursion, all-loop $n$-point planar MHV integrands [130-132] and two-loop planar $\mathrm{N}^{k} \mathrm{MHV}$ integrands [133] have been expressed in terms of only diagrams with unit leading singularities, and these naturally involve manifestly IR-finite integrals. Similar structures have now also been found beyond the planar limit [134-136], but a full proof of uniform transcendentality remains elusive. A better understanding of how the property is violated in theories with $\mathcal{N}<4$ supersymmetries may shed light on this question.

It would therefore be desirable to confirm that the minimal violation of uniform transcendentality for the finite amplitude, as defined by the IR scheme (6.1), continues for $n>4$. It will be particularly interesting to see how the two-loop IR-controlling numerators generalize for more external legs. At $n=5$ points integration of the full-color MHV amplitude should be achievable using currently available technology, given recent progress on integrated five-parton amplitudes in QCD [137-140]. Extensions to the next loop order or higher orders in $\epsilon$ are also within reach [33, 141]. Furthermore, the case of lower supersymmetry should also be explored. In the high-energy limit, a connection between superconformal symmetry and uniform transcendental weight was found for the BFKL ladder at next-to-leading logarithmic accuracy [142]. It would be interesting to see if $\mathcal{N}=1$ SQCD has a similar minimal departure from uniform transcendentality when tuned to a conformal point and if a similar relation between infrared structure and transcendental weight can be constructed in the $\mathcal{N}=1$ case.

Finally, the precise form of eq. (6.1) suggests a better interpretation may exist in the language of form factors. Each of the triangle integrals has precisely one off-shell leg, which might indicate an expectation value of some operator in $\mathcal{N}=2$ SQCD. Given that the same transcendentality properties of amplitudes are expected to carry over to form factors, as has been observed in $\mathcal{N}=4$ SYM [143-145], such an analysis may shed further light on transcendentality violations in $\mathcal{N}<4$ supersymmetric theories. 


\section{Acknowledgments}

We thank Charalampos Anastasiou, Simon Badger, Lance Dixon, Claude Duhr, Einan Gardi, Henrik Johansson, Ben Page, Alexander Penin, Oliver Schlotterer, and Leonardo Vernazza for interesting and helpful discussions. AO would also like to acknowledge the hospitality of the Galileo Galilei Institute for Theoretical Physics during the program "Amplitudes in the LHC era." AO has received funding from the European Union's Horizon 2020 research and innovation programme under the Marie Skłodowska-Curie grant agreement 746138 and ERC grant PertQCD (694712). The research of GK and GM is supported by the Swedish Research Council under grant 621-2014-5722, the Knut and Alice Wallenberg Foundation under grants KAW 2013.0235, 2018.0116, and the Ragnar Söderberg Foundation (Swedish Foundations' Starting Grant). GK has also received funding from the Knut and Alice Wallenberg Foundation under grant KAW 2018.0441, and is supported in part by the US Department of Energy under contract DE-AC02-76SF00515. BV is supported by the European Research Council under ERC grant UNISCAMP (804286).

\section{A Anomalous dimensions in $\mathcal{N}=4 \mathrm{SYM}$}

Exact four-point (and five-point) amplitudes in planar $\mathcal{N}=4 \mathrm{SYM}$ (with $G=\mathrm{SU}\left(N_{c}\right)$ ) are given by the ABDK/BDS ansatz [27, 28] which exponentiates the one-loop amplitudes evaluated to all orders in $\epsilon$ :

$$
\begin{aligned}
& \mathcal{M}_{n}^{[\mathcal{N}=4]}=\left(4 \pi \alpha_{\mathrm{s}}\right)^{\frac{n-2}{2}} \sum_{\text {perms }} \frac{1}{n} \operatorname{tr}\left[T^{a_{1}} \ldots T^{a_{n}}\right] A_{n}^{(0)}(1, \ldots, n) M_{n}(1, \ldots, n ; \epsilon)\left(1+\mathcal{O}\left(1 / N_{c}\right)\right), \\
& M_{n} \equiv 1+\sum_{L=1}^{\infty}\left(\frac{\alpha_{\mathrm{s}} S_{\epsilon}}{4 \pi} N_{c}\right)^{L} M_{n}^{(L)}(\epsilon) \\
& =\exp \left\{\sum_{L=1}^{\infty}\left(\frac{\alpha_{\mathrm{s}} S_{\epsilon}}{4 \pi} N_{c}\right)^{L}\left[f^{(L)}(\epsilon) M_{n}^{(1)}(L \epsilon)+C^{(L)}+E_{n}^{(L)}(\epsilon)\right]\right\}, \quad n=4,5,
\end{aligned}
$$

where $f^{(L)}(\epsilon)$ and $C^{(L)}$ are independent of the external kinematics, and at one loop $f^{(1)}(\epsilon)=1, C^{(1)}=E_{n}^{(1)}(\epsilon)=0$ by definition. Recall that at four points, for instance, the tree and one-loop color-ordered amplitudes are given by

$$
A_{4}^{(0)[\mathcal{N}=4]}=-\frac{i \delta^{8}(Q)}{s t} \frac{[12][34]}{\langle 12\rangle\langle 34\rangle}, \quad M_{4}^{(1)}=-s t{ }_{3}^{4} \boldsymbol{Z}_{2}^{1} .
$$

The two-loop planar amplitude is expressed as [27]

$$
M_{n}^{(2)}(\epsilon)=\frac{1}{2}\left[M_{n}^{(1)}(\epsilon)\right]^{2}-2\left(\zeta_{2}+\zeta_{3} \epsilon+\zeta_{4} \epsilon^{2}\right) M_{n}^{(1)}(2 \epsilon)+\mathcal{O}\left(\epsilon^{0}\right) .
$$

Now let us compare that with the $\mathcal{N}=4$ factorization formulae (2.25), which take the following form for the color-ordered amplitudes:

$$
\begin{aligned}
& M_{n}^{(1)}=S(\epsilon) M_{n}^{(0)}+H_{n}^{(1)}, \quad S(\epsilon)=-\frac{1}{\epsilon^{2}} \sum_{i=1}^{n}\left[1-\epsilon \log \left(\frac{-s_{i(i+1)}}{\mu^{2}}\right)\right] \\
& M_{n}^{(2)}=S(\epsilon) M_{n}^{(1)}+\left[-\frac{1}{2}[S(\epsilon)]^{2}+\frac{1}{2 N_{c}} \gamma_{K}^{(2)[\mathcal{N}=4]} S(2 \epsilon)+\frac{n}{\epsilon N_{c}^{2}} \gamma_{g}^{(2)[\mathcal{N}=4]}\right] M_{n}^{(0)}+H_{n}^{(2)},
\end{aligned}
$$


where the $N_{c}$ denominators are due to the explicit factors of $N_{c}$ in the expansion (A.1). Note that $M_{n}^{(0)} \equiv 1$, so we can equate eqs. (A.3) and (A.4b):

$$
\begin{aligned}
M_{n}^{(2)} & =\frac{1}{2}[S(\epsilon)]^{2}+S(\epsilon) H_{n}^{(1)}-2\left(\zeta_{2}+\zeta_{3} \epsilon\right) S(2 \epsilon)+\mathcal{O}\left(\epsilon^{0}\right) \\
& =\frac{1}{2}[S(\epsilon)]^{2}+S(\epsilon) H_{n}^{(1)}+\frac{1}{2 N_{c}} \gamma_{K}^{(2)[\mathcal{N}=4]} S(2 \epsilon)+\frac{n}{\epsilon N_{c}^{2}} \gamma_{g}^{(2)[\mathcal{N}=4]}+H_{n}^{(2)}
\end{aligned}
$$

Therefore, consistent with eq. (2.26), we find

$$
\gamma_{K}^{(2)[\mathcal{N}=4]}=-4 \zeta_{2} N_{c}, \quad \gamma_{g}^{(2)[\mathcal{N}=4]}=\frac{1}{2} \zeta_{3} N_{c}^{2},
$$

where we have used $S(2 \epsilon)=-n /\left(4 \epsilon^{2}\right)+\mathcal{O}\left(\epsilon^{-1}\right)$ for the latter.

\section{B Two-loop finite remainder in $\mathcal{N}=2$ SQCD}

Here we present the finite remainder function of the two-loop gluonic amplitude $\mathcal{W}_{4}^{(2) \text { fin }}$ as defined in (2.32). We split the result into two parts

$$
\mathcal{W}_{4}^{(2) \mathrm{fin}}=\mathcal{R}_{4}^{(2) \mathrm{fin}}+\left(C_{A}-T_{F} N_{f}\right) \mathcal{S}_{4}^{(2) \mathrm{fin}}
$$

where $\mathcal{R}_{4}^{(2) \text { fin }}$ denotes the remainder for the conformal theory and $\mathcal{S}_{4}^{(2) \text { fin }}$ represents corrections thereof for the generic theory (recall that $\left.\left(C_{A}-T_{F} N_{f}\right)=\beta_{0}\right)$. We present the results in terms of color-ordered building blocks in the trace basis of the gauge group $\mathrm{SU}\left(N_{c}\right)$; we denote the kinematic coefficient of $N_{c}^{i} M^{(0)}\left(1^{-}, 2^{-}, 3^{+}, 4^{+}\right) \operatorname{tr}\left(T^{a_{1}} T^{a_{2}} T^{a_{3}} T^{a_{4}}\right)$ as $W_{(--++)}^{(2)[i] \mathrm{fin}}$ and that of the double trace $N_{c}^{i} M^{(0)}\left(1^{-}, 2^{-}, 3^{+}, 4^{+}\right) \operatorname{tr}\left(T^{a_{1}} T^{a_{2}}\right) \operatorname{tr}\left(T^{a_{3}} T^{a_{4}}\right)$ as $W_{(--)(++)}^{(2)[i] \mathrm{fin}}$. Analogous notation is used for the constituent $\mathcal{R}_{4}^{(2) \text { fin }}$ and $\mathcal{S}_{4}^{(2) \text { fin }}$. The results for $\mathcal{R}_{4}^{(2) \text { fin }}$ were already obtained in ref. [40] and in the planar case in refs. [41, 44], but we list them here again for completeness. Note that, as discussed in ref. [40], the components given below form a sufficient set to reconstruct the full-color answer.

We introduce the shorthand notation $\tau=-t / s, v=-u / s$ with their logarithms being written as $\mathcal{T}=\log (\tau)$, and $\mathcal{U}=\log (v)$. Furthermore, $\operatorname{Li}_{n}(z)$ are the classical polylogarithms $[23,24]$ and $S_{n, p}(z)$ are Nielsen generalized polylogarithms (see e.g. ref. [146]). We give results in the region $s>0 ; t, u<0$, so $\mathcal{T}$ and $\mathcal{U}$ are real.

$$
\begin{aligned}
& R_{(--++)}^{(2)[2]}=\frac{\tau}{6}[ 48 \mathrm{Li}_{4}(\tau)-24(\mathcal{T}+\mathcal{U}) \mathrm{Li}_{3}(\tau)-24 \mathcal{T} \operatorname{Li}_{3}(v)+24 \mathcal{T} \mathcal{U} \operatorname{Li}_{2}(\tau)+24 \mathcal{T} \mathcal{U} \operatorname{Li}_{2}(v) \\
&-24 S_{2,2}(\tau)+\mathcal{T}^{4}-4 \mathcal{T}^{3} \mathcal{U}+18 \mathcal{T}^{2} \mathcal{U}^{2}+24 \zeta_{2} \operatorname{Li}_{2}(\tau)-12 \zeta_{2} \mathcal{T}^{2}+24 \zeta_{2} \mathcal{T U} \\
&+\left.24 \zeta_{3} \mathcal{U}-168 \zeta_{4}\right] \\
&-i \pi \frac{2 \tau}{3}\left[6 \mathrm{Li}_{3}(\tau)+6 \operatorname{Li}_{3}(v)-6 \mathcal{U} \operatorname{Li}_{2}(\tau)-6 \mathcal{U} \operatorname{Li}_{2}(v)\right. \\
&\left.-\mathcal{T}^{3}+3 \mathcal{T}^{2} \mathcal{U}-6 \mathcal{T} \mathcal{U}^{2}+6 \zeta_{2} \mathcal{U}-6 \zeta_{2} \mathcal{T}\right]+12 \zeta_{3}+\mathcal{O}(\epsilon)
\end{aligned}
$$




$$
\begin{aligned}
& R_{(--)(++)}^{(2)[1] \mathrm{fin}}=\frac{2 \tau}{3}\left[96 \mathrm{Li}_{4}(\tau)+96 \mathrm{Li}_{4}(v)-24(3 \mathcal{T}-\mathcal{U}) \mathrm{Li}_{3}(\tau)+24(\mathcal{T}-3 \mathcal{U}) \mathrm{Li}_{3}(v)\right. \\
& +24 \mathcal{T}(\mathcal{T}-\mathcal{U}) \operatorname{Li}_{2}(\tau)-24 \mathcal{U}(\mathcal{T}-\mathcal{U}) \operatorname{Li}_{2}(v) \\
& \left.+(\mathcal{T}+\mathcal{U})^{4}-24 \mathcal{T}^{2} \mathcal{U}^{2}-12 \zeta_{2}(\mathcal{T}-\mathcal{U})^{2}-654 \zeta_{4}\right] \\
& -i \pi \frac{8 \tau}{3}\left[12 \mathrm{Li}_{3}(\tau)+12 \mathrm{Li}_{3}(v)-12 \mathcal{T} \mathrm{Li}_{2}(\tau)-12 \mathcal{U} \operatorname{Li}_{2}(v)\right. \\
& \left.-(\mathcal{T}+\mathcal{U})^{3}-18 \zeta_{2}(\mathcal{T}+\mathcal{U})\right]+\mathcal{O}(\epsilon) . \\
& S_{(--++)}^{(2)[1] \mathrm{fin}}=-\frac{\tau}{6}\left[48 \mathrm{Li}_{4}(\tau)-24(\mathcal{T}+\mathcal{U}) \mathrm{Li}_{3}(\tau)-24 \mathcal{T} \operatorname{Li}_{3}(v)+24 \mathcal{T} \mathcal{U} \operatorname{Li}_{2}(\tau)+24 \mathcal{T} \mathcal{U} \operatorname{Li}_{2}(v)\right. \\
& \left.-24 S_{2,2}(\tau)+\mathcal{T}^{4}-4 \mathcal{T}^{3} U+18 \mathcal{T}^{2} \mathcal{U}^{2}+24 \zeta_{2} \operatorname{Li}_{2}(\tau)-12 \zeta_{2} \mathcal{T}^{2}+24 \zeta_{2} \mathcal{T U}+24 \zeta_{3} \mathcal{U}-168 \zeta_{4}\right] \\
& +i \pi \frac{2 \tau}{3}\left[6 \operatorname{Li}_{3}(\tau)+6 \operatorname{Li}_{3}(v)-6 \mathcal{U} \operatorname{Li}_{2}(\tau)-6 \mathcal{U} \operatorname{Li}_{2}(v)-\mathcal{T}^{3}+3 \mathcal{T}^{2} \mathcal{U}-6 \mathcal{T} \mathcal{U}^{2}-6 \zeta_{2}(\mathcal{T}-\mathcal{U})\right] \\
& -\frac{1}{3}\left[6 \operatorname{Li}_{3}(\tau)-6 \mathcal{T} \operatorname{Li}_{2}(\tau)-\mathcal{T}^{3}-3 \mathcal{T}^{2} \mathcal{U}+24 \zeta_{2} \mathcal{T}+12 \zeta_{3}\right] \\
& +i \pi\left[2 \mathrm{Li}_{2}(\tau)+\mathcal{T}^{2}+2 \mathcal{T} \mathcal{U}-4 \zeta_{2}\right]+\mathcal{O}(\epsilon), \\
& S_{(--)(++)}^{(2)[0] \mathrm{fin}}=-\frac{2}{3 v}\left[12 \tau v \operatorname{Li}_{3}(\tau)+12 \tau v \operatorname{Li}_{3}(v)-12 \tau v \mathcal{T} \operatorname{Li}_{2}(\tau)-12 \tau v \mathcal{U} \operatorname{Li}_{2}(v)\right. \\
& \left.-2 \tau^{2} \mathcal{T}^{3}-3\left(1-2 \tau^{2}\right) \mathcal{T}^{2} \mathcal{U}+3(1-2 \tau-2 \tau v) \mathcal{T} \mathcal{U}^{2}-2 v^{2} \mathcal{U}^{3}-6(\tau-3 v) \zeta_{2} \mathcal{T}-6\left(1-4 \tau^{2}\right) \zeta_{2} \mathcal{U}\right] \\
& -i \pi \frac{2}{v}\left[2 \tau v(\mathcal{T}-\mathcal{U})^{2}-\mathcal{T}^{2}-\mathcal{U}^{2}-4(1-3 \tau v) \zeta_{2}\right]+\mathcal{O}(\epsilon), \\
& S_{(--++)}^{(2)[-1] \mathrm{fin}}=-\frac{\tau}{12}\left[48 \mathrm{Li}_{4}(\tau)-24(\mathcal{T}+\mathcal{U}) \mathrm{Li}_{3}(\tau)-24 \mathcal{T} \operatorname{Li}_{3}(v)\right. \\
& +24 \mathcal{T} \mathcal{U} \operatorname{Li}_{2}(\tau)+24 \mathcal{T} \mathcal{U} \operatorname{Li}_{2}(v)-24 S_{2,2}(\tau)+\mathcal{T}^{4}-4 \mathcal{T}^{3} \mathcal{U}+18 \mathcal{T}^{2} \mathcal{U}^{2} \\
& \left.+24 \zeta_{2} \operatorname{Li}_{2}(\tau)-12 \zeta_{2} \mathcal{T}^{2}+24 \zeta_{2} \mathcal{T} \mathcal{U}+24 \zeta_{3} \mathcal{U}-168 \zeta_{4}\right] \\
& +i \pi \frac{\tau}{3}\left[6 \operatorname{Li}_{3}(\tau)+6 \operatorname{Li}_{3}(v)-6 \mathcal{U} \operatorname{Li}_{2}(\tau)-6 \mathcal{U} \operatorname{Li}_{2}(v)\right. \\
& \left.-\mathcal{T}^{3}+3 \mathcal{T}^{2} \mathcal{U}-6 \mathcal{T} \mathcal{U}^{2}-6 \zeta_{2}(\mathcal{T}-\mathcal{U})\right]-6 \zeta_{3}+\mathcal{O}(\epsilon) . \\
& R_{(-+-+)}^{(2)[2]}=\frac{\tau}{6 v^{2}} \mathcal{T}^{2}\left(\mathcal{T}^{2}-24 \zeta_{2}\right)+i \pi \frac{2 \tau}{3 v^{2}} \mathcal{T}^{3}+12 \zeta_{3}+\mathcal{O}(\epsilon), \\
& R_{(-+)(-+)}^{(2)[1] \mathrm{fin}}=\frac{2 \tau}{3 v^{2}}\left[48 \operatorname{Li}_{4}(\tau)-24 \mathcal{T} \operatorname{Li}_{3}(\tau)-24 S_{2,2}(\tau)+\mathcal{T}^{4}\right. \\
& \left.+24 \zeta_{2} \operatorname{Li}_{2}(\tau)-84 \zeta_{2} \mathcal{T}^{2}+24 \zeta_{3} \mathcal{T}-102 \zeta_{4}\right]+i \pi \frac{16 \tau}{3 v^{2}} \mathcal{T}\left[\mathcal{T}^{2}-3 \zeta_{2}\right] \\
& -\frac{16 \tau}{v^{2}}\left[\tau \operatorname{Li}_{3}(\tau)+v \operatorname{Li}_{3}(v)-\tau \mathcal{T} \operatorname{Li}_{2}(\tau)-v \mathcal{U} \operatorname{Li}_{2}(v)-5 \tau \zeta_{2} \mathcal{T}-5 v \zeta_{2} \mathcal{U}-\zeta_{3}\right] \\
& +i \pi \frac{8 \tau}{v^{2}}\left[2(\tau-v) \mathrm{Li}_{2}(\tau)-\tau \mathcal{T}^{2}-2 v \mathcal{T} \mathcal{U}+v \mathcal{U}^{2}-2 \tau \zeta_{2}\right]+\mathcal{O}(\epsilon) . \\
& S_{(-+-+)}^{(2)[1] \mathrm{fin}}=\frac{\tau}{4 v^{2}} \mathcal{T}^{2}\left[\mathcal{T}^{2}-32 \zeta_{2}\right]+i \pi \frac{\tau}{v^{2}} \mathcal{T}\left[\mathcal{T}^{2}-4 \zeta_{2}\right] \\
& -\frac{1}{3 v}\left[6 v \operatorname{Li}_{3}(\tau)-6 v \mathcal{T} \operatorname{Li}_{2}(\tau)-(1+2 \tau) \mathcal{T}^{3}-3(1-\tau) \mathcal{T}^{2} \mathcal{U}+24(1+\tau) \zeta_{2} \mathcal{T}+12 v \zeta_{3}\right] \\
& +i \pi \frac{1}{v}\left[2 v \operatorname{Li}_{2}(\tau)+(1+2 \tau) \mathcal{T}^{2}+2 v \mathcal{T} \mathcal{U}-4 \zeta_{2}\right]+\mathcal{O}(\epsilon),
\end{aligned}
$$




$$
\begin{aligned}
& S_{(-+)(-+)}^{(2)[0] \mathrm{fin}}=\frac{\tau}{3 v^{2}}[ 72 \mathrm{Li}_{4}(\tau)-24(2 \mathcal{T}+\mathcal{U}) \mathrm{Li}_{3}(\tau)-48 \mathcal{T} \operatorname{Li}_{3}(v) \\
&+12 \mathcal{T}(\mathcal{T}+2 \mathcal{U}) \operatorname{Li}_{2}(\tau)+48 \mathcal{T} \mathcal{L} \operatorname{Li}_{2}(v)-60 S_{2,2}(\tau)+4 \mathcal{T}^{3} \mathcal{U}+24 \mathcal{T}^{2} \mathcal{U}^{2} \\
&\left.+84 \zeta_{2} \operatorname{Li}_{2}(\tau)-54 \zeta_{2} \mathcal{T}^{2}+72 \zeta_{2} \mathcal{T} \mathcal{U}+24 \zeta_{3}(2 \mathcal{T}+\mathcal{U})-267 \zeta_{4}\right] \\
&- i \pi \frac{4 \tau}{3 v^{2}}\left[3 \operatorname{Li}_{3}(\tau)+12 \operatorname{Li}_{3}(v)-6 \mathcal{U} \operatorname{Li}_{2}(\tau)-12 \mathcal{U} \operatorname{Li}_{2}(v)-\mathcal{T}^{3}-6 \mathcal{T} \mathcal{U}^{2}-3 \zeta_{2}(\mathcal{T}-2 \mathcal{U})-3 \zeta_{3}\right] \\
&+ \frac{2}{3 v^{2}}\left[6 \tau(1+v) \operatorname{Li}_{3}(\tau)-6 \tau v \operatorname{Li}_{3}(v)-6 \tau(1+v) \mathcal{T} \operatorname{Li}_{2}(\tau)+6 \tau v \mathcal{U} \operatorname{Li}_{2}(v)-2 \tau^{2} \mathcal{T}^{3}\right. \\
&\left.+3 v^{2} \mathcal{T U}(\mathcal{T}-\mathcal{U})+2 v^{2} \mathcal{U}^{3}-6\left(3+2 \tau-4 \tau^{2}\right) \zeta_{2} \mathcal{T}+6\left(1+3 \tau-4 \tau^{2}\right) \zeta_{2} \mathcal{U}-6 \tau \zeta_{3}\right] \\
&- \frac{2 i \pi}{v^{2}}\left[2 \tau(3-2 \tau) \operatorname{Li}_{2}(\tau)-\left(1-2 \tau^{2}\right) \mathcal{T}^{2}+2 \tau v \mathcal{T} \mathcal{U}+v(\tau-v) \mathcal{U}^{2}-2\left(2-\tau^{2}\right) \zeta_{2}\right]+\mathcal{O}(\epsilon), \\
& S_{(-+-+)}^{(2)[-1] \mathrm{fin}}=-\frac{\tau}{12 v^{2}} \mathcal{T}^{2}\left[\mathcal{T}^{2}-24 \zeta_{2}\right]-i \pi \frac{\tau}{3 v^{2}} \mathcal{T}^{3}-6 \zeta_{3}+\mathcal{O}(\epsilon) .
\end{aligned}
$$

Open Access. This article is distributed under the terms of the Creative Commons Attribution License (CC-BY 4.0), which permits any use, distribution and reproduction in any medium, provided the original author(s) and source are credited.

\section{References}

[1] E. Gardi, Recent progress on infrared singularities, PoS (RADCOR2017) 037 (2018) [arXiv: 1801.03174] [INSPIRE].

[2] H. Elvang and Y.-t. Huang, Scattering Amplitudes in Gauge Theory and Gravity, Cambridge University Press (2015) [INSPIRE].

[3] L.J. Dixon, Scattering amplitudes: the most perfect microscopic structures in the universe, J. Phys. A 44 (2011) 454001 [arXiv:1105.0771] [InSPIRE].

[4] L.J. Dixon, Calculating scattering amplitudes efficiently, in $Q C D$ and beyond. Proceedings, Theoretical Advanced Study Institute in Elementary Particle Physics, TASI-95, Boulder, U.S.A., 4-30 June 1995, pp. 539-584 (1996) [hep-ph/9601359] [INSPIRE].

[5] R. Akhoury, Mass Divergences of Wide Angle Scattering Amplitudes, Phys. Rev. D 19 (1979) 1250 [INSPIRE].

[6] A. Sen, Asymptotic Behavior of the Wide Angle On-Shell Quark Scattering Amplitudes in Nonabelian Gauge Theories, Phys. Rev. D 28 (1983) 860 [INSPIRE].

[7] G.F. Sterman and M.E. Tejeda-Yeomans, Multiloop amplitudes and resummation, Phys. Lett. B 552 (2003) 48 [hep-ph/0210130] [INSPIRE].

[8] S.M. Aybat, L.J. Dixon and G.F. Sterman, The Two-loop anomalous dimension matrix for soft gluon exchange, Phys. Rev. Lett. 97 (2006) 072001 [hep-ph/0606254] [INSPIRE].

[9] S.M. Aybat, L.J. Dixon and G.F. Sterman, The Two-loop soft anomalous dimension matrix and resummation at next-to-next-to leading pole, Phys. Rev. D 74 (2006) 074004 [hep-ph/0607309] [INSPIRE].

[10] E. Gardi and L. Magnea, Factorization constraints for soft anomalous dimensions in $Q C D$ scattering amplitudes, JHEP 03 (2009) 079 [arXiv:0901.1091] [INSPIRE].

[11] E. Gardi and L. Magnea, Infrared singularities in QCD amplitudes, Nuovo Cim. C32N5-6 (2009) 137 [arXiv: 0908.3273] [INSPIRE]. 
[12] T. Becher and M. Neubert, Infrared singularities of scattering amplitudes in perturbative QCD, Phys. Rev. Lett. 102 (2009) 162001 [Erratum ibid. 111 (2013) 199905] [arXiv:0901.0722] [INSPIRE].

[13] T. Becher and M. Neubert, On the Structure of Infrared Singularities of Gauge-Theory Amplitudes, JHEP 06 (2009) 081 [Erratum ibid. 11 (2013) 024] [arXiv:0903.1126] [INSPIRE].

[14] I. Moult, I.W. Stewart, G. Vita and H.X. Zhu, The Soft Quark Sudakov, arXiv:1910.14038 [INSPIRE].

[15] S. Catani, The Singular behavior of QCD amplitudes at two loop order, Phys. Lett. B 427 (1998) 161 [hep-ph/9802439] [INSPIRE].

[16] S. Badger, G. Mogull and T. Peraro, Local integrands for two-loop all-plus Yang-Mills amplitudes, JHEP 08 (2016) 063 [arXiv:1606.02244] [INSPIRE].

[17] S. Badger, G. Mogull and T. Peraro, Local integrands for two-loop QCD amplitudes, PoS (LL2016) 006 (2016) [arXiv: 1607.00311] [INSPIRE].

[18] D.C. Dunbar and W.B. Perkins, Two-loop five-point all plus helicity Yang-Mills amplitude, Phys. Rev. D 93 (2016) 085029 [arXiv: 1603.07514] [INSPIRE].

[19] D.C. Dunbar, G.R. Jehu and W.B. Perkins, The two-loop n-point all-plus helicity amplitude, Phys. Rev. D 93 (2016) 125006 [arXiv:1604.06631] [INSPIRE].

[20] D.C. Dunbar, G.R. Jehu and W.B. Perkins, Two-loop six gluon all plus helicity amplitude, Phys. Rev. Lett. 117 (2016) 061602 [arXiv:1605.06351] [INSPIRE].

[21] D.C. Dunbar, J.H. Godwin, G.R. Jehu and W.B. Perkins, Analytic all-plus-helicity gluon amplitudes in QCD, Phys. Rev. D 96 (2017) 116013 [arXiv:1710.10071] [InSPIRE].

[22] D.C. Dunbar, J.H. Godwin, W.B. Perkins and J.M.W. Strong, Color Dressed Unitarity and Recursion for Yang-Mills Two-Loop All-Plus Amplitudes, arXiv:1911.06547 [INSPIRE].

[23] A.B. Goncharov, Multiple polylogarithms, cyclotomy and modular complexes, Math. Res. Lett. 5 (1998) 497 [arXiv:1105.2076] [INSPIRE].

[24] A.B. Goncharov, Multiple polylogarithms and mixed Tate motives, math/0103059.

[25] N. Arkani-Hamed, F. Cachazo and J. Kaplan, What is the Simplest Quantum Field Theory?, JHEP 09 (2010) 016 [arXiv:0808.1446] [INSPIRE].

[26] Z. Bern, J.S. Rozowsky and B. Yan, Two loop four gluon amplitudes in $N=4$ superYang-Mills, Phys. Lett. B 401 (1997) 273 [hep-ph/9702424] [INSPIRE].

[27] C. Anastasiou, Z. Bern, L.J. Dixon and D.A. Kosower, Planar amplitudes in maximally supersymmetric Yang-Mills theory, Phys. Rev. Lett. 91 (2003) 251602 [hep-th/0309040] [INSPIRE].

[28] Z. Bern, L.J. Dixon and V.A. Smirnov, Iteration of planar amplitudes in maximally supersymmetric Yang-Mills theory at three loops and beyond, Phys. Rev. D 72 (2005) 085001 [hep-th/0505205] [INSPIRE].

[29] S.G. Naculich, H. Nastase and H.J. Schnitzer, Subleading-color contributions to gluon-gluon scattering in $N=4$ SYM theory and relations to $N=8$ supergravity, JHEP 11 (2008) 018 [arXiv:0809.0376] [INSPIRE].

[30] V. Del Duca, C. Duhr and V.A. Smirnov, The Two-Loop Hexagon Wilson Loop in $N=4$ SYM, JHEP 05 (2010) 084 [arXiv:1003.1702] [INSPIRE]. 
[31] A.B. Goncharov, M. Spradlin, C. Vergu and A. Volovich, Classical Polylogarithms for Amplitudes and Wilson Loops, Phys. Rev. Lett. 105 (2010) 151605 [arXiv:1006.5703] [INSPIRE].

[32] J. Golden, M.F. Paulos, M. Spradlin and A. Volovich, Cluster Polylogarithms for Scattering Amplitudes, J. Phys. A 47 (2014) 474005 [arXiv: 1401.6446] [InSPIRE].

[33] J.M. Henn and B. Mistlberger, Four-Gluon Scattering at Three Loops, Infrared Structure and the Regge Limit, Phys. Rev. Lett. 117 (2016) 171601 [arXiv:1608.00850] [InSPIRE].

[34] J. Drummond, J. Foster, Ö. Gürdoğan and G. Papathanasiou, Cluster adjacency and the four-loop NMHV heptagon, JHEP 03 (2019) 087 [arXiv:1812.04640] [INSPIRE].

[35] S. Abreu, L.J. Dixon, E. Herrmann, B. Page and M. Zeng, The two-loop five-point amplitude in $\mathcal{N}=4$ super-Yang-Mills theory, Phys. Rev. Lett. 122 (2019) 121603 [arXiv: 1812.08941] [INSPIRE].

[36] D. Chicherin, T. Gehrmann, J.M. Henn, P. Wasser, Y. Zhang and S. Zoia, Analytic result for a two-loop five-particle amplitude, Phys. Rev. Lett. 122 (2019) 121602 [arXiv: 1812.11057] [INSPIRE].

[37] S. Caron-Huot, L.J. Dixon, F. Dulat, M. von Hippel, A.J. McLeod and G. Papathanasiou, Six-Gluon amplitudes in planar $\mathcal{N}=4$ super-Yang-Mills theory at six and seven loops, JHEP 08 (2019) 016 [arXiv:1903.10890] [INSPIRE].

[38] A.V. Kotikov and L.N. Lipatov, DGLAP and BFKL evolution equations in the $N=4$ supersymmetric gauge theory, in 35th Annual Winter School on Nuclear and Particle Physics, Repino, Russia, 19-25 February 2001 (2001) [hep-ph/0112346] [INSPIRE].

[39] A.V. Kotikov and L.N. Lipatov, DGLAP and BFKL equations in the $N=4$ supersymmetric gauge theory, Nucl. Phys. B 661 (2003) 19 [Erratum ibid. B 685 (2004) 405] [hep-ph/0208220] [INSPIRE].

[40] C. Duhr, H. Johansson, G. Kälin, G. Mogull and B. Verbeek, The Full-Color Two-Loop Four-Gluon Amplitude in $\mathcal{N}=2$ Super-QCD, Phys. Rev. Lett. 123 (2019) 241601 [arXiv: 1904.05299] [INSPIRE].

[41] L. Dixon, Gluon Scattering in N=4 super-Yang-Mills Theory from Weak to Strong Coupling, talk, based on work with D. Kosower and C. Vergu, at Workshop on Gauge and String Theory at ETH Zürich, Switzerland, 2-4 July 2008

[http://conf.itp.phys.ethz.ch/gaugestring08/].

[42] R. Andree and D. Young, Wilson Loops in $N=2$ Superconformal Yang-Mills Theory, JHEP 09 (2010) 095 [arXiv: 1007.4923] [INSPIRE].

[43] M. Leoni, A. Mauri and A. Santambrogio, Four-point amplitudes in $\mathcal{N}=2$ SCQCD, JHEP 09 (2014) 017 [Erratum ibid. 02 (2015) 022] [arXiv:1406.7283] [INSPIRE].

[44] M. Leoni, A. Mauri and A. Santambrogio, On the amplitude/Wilson loop duality in $N=2$ SCQCD, Phys. Lett. B 747 (2015) 325 [arXiv:1502.07614] [INSPIRE].

[45] H. Johansson, G. Kälin and G. Mogull, Two-loop supersymmetric QCD and half-maximal supergravity amplitudes, JHEP 09 (2017) 019 [arXiv:1706.09381] [INSPIRE].

[46] G. Kälin, G. Mogull and A. Ochirov, Two-loop $\mathcal{N}=2$ SQCD amplitudes with external matter from iterated cuts, JHEP 07 (2019) 120 [arXiv: 1811.09604] [INSPIRE]. 
[47] Z. Bern, J.J.M. Carrasco and H. Johansson, New Relations for Gauge-Theory Amplitudes, Phys. Rev. D 78 (2008) 085011 [arXiv:0805.3993] [InSPIRE].

[48] Z. Bern, J.J.M. Carrasco and H. Johansson, Perturbative Quantum Gravity as a Double Copy of Gauge Theory, Phys. Rev. Lett. 105 (2010) 061602 [arXiv:1004.0476] [INSPIRE].

[49] H. Johansson and A. Ochirov, Pure Gravities via Color-Kinematics Duality for Fundamental Matter, JHEP 11 (2015) 046 [arXiv: 1407.4772] [INSPIRE].

[50] H. Johansson and A. Ochirov, Color-Kinematics Duality for QCD Amplitudes, JHEP 01 (2016) 170 [arXiv: 1507.00332] [INSPIRE].

[51] Z. Bern, J.J. Carrasco, M. Chiodaroli, H. Johansson and R. Roiban, The Duality Between Color and Kinematics and its Applications, arXiv:1909.01358 [INSPIRE].

[52] S. Badger, G. Mogull, A. Ochirov and D. O'Connell, A Complete Two-Loop, Five-Gluon Helicity Amplitude in Yang-Mills Theory, JHEP 10 (2015) 064 [arXiv:1507.08797] [INSPIRE].

[53] A. Ochirov and B. Page, Full Colour for Loop Amplitudes in Yang-Mills Theory, JHEP 02 (2017) 100 [arXiv: 1612.04366] [inSPIRE].

[54] A. Ochirov and B. Page, Multi-Quark Colour Decompositions from Unitarity, JHEP 10 (2019) 058 [arXiv: 1908.02695] [InSPIRE].

[55] Ø. Almelid, C. Duhr and E. Gardi, Three-loop corrections to the soft anomalous dimension in multileg scattering, Phys. Rev. Lett. 117 (2016) 172002 [arXiv:1507.00047] [INSPIRE].

[56] E. Gardi, Ø. Almelid and C. Duhr, Long-distance singularities in multi-leg scattering amplitudes, PoS (LL2016) 058 (2016) [arXiv: 1606 . 05697] [INSPIRE].

[57] Ø. Almelid, C. Duhr, E. Gardi, A. McLeod and C.D. White, Bootstrapping the QCD soft anomalous dimension, JHEP 09 (2017) 073 [arXiv: 1706.10162] [INSPIRE].

[58] C. Gnendiger, A. Signer and D. Stöckinger, The infrared structure of QCD amplitudes and $H \rightarrow g g$ in FDH and DRED, Phys. Lett. B 733 (2014) 296 [arXiv:1404.2171] [INSPIRE].

[59] S. Catani and M.H. Seymour, The Dipole formalism for the calculation of QCD jet cross-sections at next-to-leading order, Phys. Lett. B 378 (1996) 287 [hep-ph/9602277] [INSPIRE].

[60] Z. Bern, L.J. Dixon and D.A. Kosower, $N=4$ super-Yang-Mills theory, $Q C D$ and collider physics, Comptes Rendus Physique 5 (2004) 955 [hep-th/0410021] [INSPIRE].

[61] Z. Bern, A. De Freitas and L.J. Dixon, Two loop helicity amplitudes for gluon-gluon scattering in QCD and supersymmetric Yang-Mills theory, JHEP 03 (2002) 018 [hep-ph/0201161] [INSPIRE].

[62] Z. Bern, A. De Freitas and L.J. Dixon, Two loop helicity amplitudes for quark gluon scattering in QCD and gluino gluon scattering in supersymmetric Yang-Mills theory, JHEP 06 (2003) 028 [Erratum ibid. 04 (2014) 112] [hep-ph/0304168] [INSPIRE].

[63] S. Caron-Huot, E. Gardi and L. Vernazza, Two-parton scattering in the high-energy limit, JHEP 06 (2017) 016 [arXiv:1701.05241] [InSPIRE].

[64] G. 't Hooft and M.J.G. Veltman, Regularization and Renormalization of Gauge Fields, Nucl. Phys. B 44 (1972) 189 [InSPIRE].

[65] J.C. Collins, Renormalization, Cambridge Monographs on Mathematical Physics, vol. 26, Cambridge University Press (1986) [INSPIRE]. 
[66] W. Siegel, Supersymmetric Dimensional Regularization via Dimensional Reduction, Phys. Lett. 84B (1979) 193 [INSPIRE].

[67] Z. Bern and D.A. Kosower, The Computation of loop amplitudes in gauge theories, Nucl. Phys. B 379 (1992) 451 [inSPIRE].

[68] Z. Bern, A. De Freitas, L.J. Dixon and H.L. Wong, Supersymmetric regularization, two loop QCD amplitudes and coupling shifts, Phys. Rev. D 66 (2002) 085002 [hep-ph/0202271] [INSPIRE].

[69] C. Gnendiger et al., To d, or not to d: recent developments and comparisons of regularization schemes, Eur. Phys. J. C 77 (2017) 471 [arXiv: 1705.01827] [InSPIRE].

[70] W.B. Kilgore, Regularization Schemes and Higher Order Corrections, Phys. Rev. D 83 (2011) 114005 [arXiv:1102.5353] [INSPIRE].

[71] R. van Damme and G. 't Hooft, Breakdown of Unitarity in the Dimensional Reduction Scheme, Phys. Lett. 150B (1985) 133 [InSPIRE].

[72] I. Jack, D.R.T. Jones and K.L. Roberts, Dimensional reduction in nonsupersymmetric theories, Z. Phys. C 62 (1994) 161 [hep-ph/9310301] [INSPIRE].

[73] I. Jack, D.R.T. Jones and K.L. Roberts, Equivalence of dimensional reduction and dimensional regularization, Z. Phys. C 63 (1994) 151 [hep-ph/9401349] [INSPIRE].

[74] S. Moch, J.A.M. Vermaseren and A. Vogt, Three-loop results for quark and gluon form-factors, Phys. Lett. B 625 (2005) 245 [hep-ph/0508055] [INSPIRE].

[75] T. Gehrmann, E.W.N. Glover, T. Huber, N. Ikizlerli and C. Studerus, Calculation of the quark and gluon form factors to three loops in QCD, JHEP 06 (2010) 094 [arXiv: 1004.3653] [INSPIRE].

[76] T. Gehrmann and E. Remiddi, Differential equations for two loop four point functions, Nucl. Phys. B 580 (2000) 485 [hep-ph/9912329] [INSPIRE].

[77] S. Kachru and E. Silverstein, 4-D conformal theories and strings on orbifolds, Phys. Rev. Lett. 80 (1998) 4855 [hep-th/9802183] [INSPIRE].

[78] A.E. Lawrence, N. Nekrasov and C. Vafa, On conformal field theories in four-dimensions, Nucl. Phys. B 533 (1998) 199 [hep-th/9803015] [INSPIRE].

[79] M. Bershadsky, Z. Kakushadze and C. Vafa, String expansion as large $N$ expansion of gauge theories, Nucl. Phys. B 523 (1998) 59 [hep-th/9803076] [INSPIRE].

[80] M. Bershadsky and A. Johansen, Large $N$ limit of orbifold field theories, Nucl. Phys. B 536 (1998) 141 [hep-th/9803249] [INSPIRE].

[81] M. Chiodaroli, Q. Jin and R. Roiban, Color/kinematics duality for general abelian orbifolds of $N=4$ super Yang-Mills theory, JHEP 01 (2014) 152 [arXiv:1311.3600] [INSPIRE].

[82] V.A. Novikov, M.A. Shifman, A.I. Vainshtein and V.I. Zakharov, Exact Gell-Mann-Low Function of Supersymmetric Yang-Mills Theories from Instanton Calculus, Nucl. Phys. B 229 (1983) 381 [INSPIRE].

[83] N. Seiberg, Supersymmetry and Nonperturbative $\beta$-functions, Phys. Lett. B 206 (1988) 75 [INSPIRE].

[84] E. Pomoni, Integrability in $N=2$ superconformal gauge theories, Nucl. Phys. B 893 (2015) 21 [arXiv: 1310.5709] [INSPIRE]. 
[85] V. Mitev and E. Pomoni, Exact effective couplings of four dimensional gauge theories with $\mathcal{N}=2$ supersymmetry, Phys. Rev. D 92 (2015) 125034 [arXiv:1406.3629] [INSPIRE].

[86] V. Mitev and E. Pomoni, Exact Bremsstrahlung and Effective Couplings, JHEP 06 (2016) 078 [arXiv: 1511.02217] [INSPIRE].

[87] Z. Bern, L.J. Dixon, D.C. Dunbar and D.A. Kosower, One loop $n$ point gauge theory amplitudes, unitarity and collinear limits, Nucl. Phys. B 425 (1994) 217 [hep-ph/9403226] [INSPIRE].

[88] Z. Bern, L.J. Dixon, D.C. Dunbar and D.A. Kosower, Fusing gauge theory tree amplitudes into loop amplitudes, Nucl. Phys. B 435 (1995) 59 [hep-ph/9409265] [InSPIRE].

[89] R. Britto, F. Cachazo and B. Feng, Generalized unitarity and one-loop amplitudes in $N=4$ super-Yang-Mills, Nucl. Phys. B 725 (2005) 275 [hep-th/0412103] [INSPIRE].

[90] W.T. Giele, Z. Kunszt and K. Melnikov, Full one-loop amplitudes from tree amplitudes, JHEP 04 (2008) 049 [arXiv:0801.2237] [InSPIRE].

[91] C. Cheung and D. O'Connell, Amplitudes and Spinor-Helicity in Six Dimensions, JHEP 07 (2009) 075 [arXiv: 0902.0981] [InSPIRE].

[92] Z. Bern, J.J. Carrasco, T. Dennen, Y.-t. Huang and H. Ita, Generalized Unitarity and Six-Dimensional Helicity, Phys. Rev. D 83 (2011) 085022 [arXiv:1010.0494] [InSPIRE].

[93] B. de Wit, Supergravity, in Unity from duality: Gravity, gauge theory and strings. Proceedings, NATO Advanced Study Institute, Euro Summer School, 76th session, Les Houches, France, 30 July-31 August 2001, pp. 1-135 (2002) [hep-th/0212245] [INSPIRE].

[94] R. Boels, Covariant representation theory of the Poincaré algebra and some of its extensions, JHEP 01 (2010) 010 [arXiv:0908.0738] [INSPIRE].

[95] T. Dennen, Y.-t. Huang and W. Siegel, Supertwistor space for $6 D$ maximal super Yang-Mills, JHEP 04 (2010) 127 [arXiv: 0910.2688] [INSPIRE].

[96] H. Elvang, Y.-t. Huang and C. Peng, On-shell superamplitudes in $N<4$ SYM, JHEP 09 (2011) 031 [arXiv: 1102.4843] [INSPIRE].

[97] Y.-t. Huang, Non-Chiral S-matrix of $N=4$ Super Yang-Mills, arXiv:1104.2021 [INSPIRE].

[98] A. De Freitas and Z. Bern, Two-loop helicity amplitudes for quark-quark scattering in QCD and gluino-gluino scattering in supersymmetric Yang-Mills theory, JHEP 09 (2004) 039 [hep-ph/0409007] [INSPIRE].

[99] M. Beneke and V.A. Smirnov, Asymptotic expansion of Feynman integrals near threshold, Nucl. Phys. B 522 (1998) 321 [hep-ph/9711391] [INSPIRE].

[100] V.A. Smirnov, Applied asymptotic expansions in momenta and masses, Springer Tracts Mod. Phys. 177 (2002) 1 [INSPIRE].

[101] B. Jantzen, Foundation and generalization of the expansion by regions, JHEP 12 (2011) 076 [arXiv:1111.2589] [INSPIRE].

[102] T.Y. Semenova, A.V. Smirnov and V.A. Smirnov, On the status of expansion by regions, Eur. Phys. J. C 79 (2019) 136 [arXiv:1809.04325] [INSPIRE].

[103] C. Anastasiou and G. Sterman, Removing infrared divergences from two-loop integrals, JHEP 07 (2019) 056 [arXiv:1812.03753] [INSPIRE]. 
[104] Z. Nagy and D.E. Soper, General subtraction method for numerical calculation of one loop QCD matrix elements, JHEP 09 (2003) 055 [hep-ph/0308127] [INSPIRE].

[105] V. Del Duca, L.J. Dixon and F. Maltoni, New color decompositions for gauge amplitudes at tree and loop level, Nucl. Phys. B 571 (2000) 51 [hep-ph/9910563] [INSPIRE].

[106] J.J.M. Carrasco, M. Chiodaroli, M. Günaydin and R. Roiban, One-loop four-point amplitudes in pure and matter-coupled $\mathcal{N} \leq 4$ supergravity, JHEP 03 (2013) 056 [arXiv:1212.1146] [INSPIRE].

[107] Z. Bern, S. Davies, T. Dennen, Y.-t. Huang and J. Nohle, Color-Kinematics Duality for Pure Yang-Mills and Gravity at One and Two Loops, Phys. Rev. D 92 (2015) 045041 [arXiv:1303.6605] [INSPIRE].

[108] J. Nohle, Color-Kinematics Duality in One-Loop Four-Gluon Amplitudes with Matter, Phys. Rev. D 90 (2014) 025020 [arXiv: 1309.7416] [INSPIRE].

[109] A. Ochirov and P. Tourkine, BCJ duality and double copy in the closed string sector, JHEP 05 (2014) 136 [arXiv: 1312.1326] [INSPIRE].

[110] S. Caron-Huot and K.J. Larsen, Uniqueness of two-loop master contours, JHEP 10 (2012) 026 [arXiv: 1205.0801] [INSPIRE].

[111] A.V. Smirnov and M.N. Tentyukov, Feynman Integral Evaluation by a Sector decomposiTion Approach (FIESTA), Comput. Phys. Commun. 180 (2009) 735 [arXiv: 0807.4129] [INSPIRE].

[112] A.V. Smirnov, FIESTA 3: cluster-parallelizable multiloop numerical calculations in physical regions, Comput. Phys. Commun. 185 (2014) 2090 [arXiv:1312.3186] [InSPIRE].

[113] A.V. Smirnov, FIESTA4: Optimized Feynman integral calculations with GPU support, Comput. Phys. Commun. 204 (2016) 189 [arXiv:1511.03614] [inSPIRE].

[114] C. Anastasiou, E.W.N. Glover and C. Oleari, The two-loop scalar and tensor pentabox graph with light-like legs, Nucl. Phys. B 575 (2000) 416 [Erratum ibid. B 585 (2000) 763] [hep-ph/9912251] [INSPIRE].

[115] C. Anastasiou, Two-loop integrals and QCD scattering, Ph.D. Thesis, Durham University (2001) [http://etheses.dur.ac.uk/4385/].

[116] O.V. Tarasov, Connection between Feynman integrals having different values of the space-time dimension, Phys. Rev. D 54 (1996) 6479 [hep-th/9606018] [INSPIRE].

[117] R.N. Lee, Space-time dimensionality D as complex variable: Calculating loop integrals using dimensional recurrence relation and analytical properties with respect to D, Nucl. Phys. B 830 (2010) 474 [arXiv: 0911. 0252] [INSPIRE].

[118] R.N. Lee, Presenting LiteRed: a tool for the Loop InTEgrals REDuction, arXiv:1212.2685 [INSPIRE].

[119] K.G. Chetyrkin and F.V. Tkachov, Integration by Parts: The Algorithm to Calculate -functions in 4 Loops, Nucl. Phys. B 192 (1981) 159 [INSPIRE].

[120] F.V. Tkachov, A Theorem on Analytical Calculability of Four Loop Renormalization Group Functions, Phys. Lett. 100B (1981) 65 [InSPIRE].

[121] V.A. Smirnov and O.L. Veretin, Analytical results for dimensionally regularized massless on-shell double boxes with arbitrary indices and numerators, Nucl. Phys. B 566 (2000) 469 [hep-ph/9907385] [INSPIRE]. 
[122] J.B. Tausk, Nonplanar massless two loop Feynman diagrams with four on-shell legs, Phys. Lett. B 469 (1999) 225 [hep-ph/9909506] [INSPIRE].

[123] C. Anastasiou, T. Gehrmann, C. Oleari, E. Remiddi and J.B. Tausk, The Tensor reduction and master integrals of the two loop massless crossed box with lightlike legs, Nucl. Phys. B 580 (2000) 577 [hep-ph/0003261] [INSPIRE].

[124] T. Gehrmann, T. Huber and D. Maître, Two-loop quark and gluon form-factors in dimensional regularisation, Phys. Lett. B 622 (2005) 295 [hep-ph/0507061] [INSPIRE].

[125] J.M. Henn, Multiloop integrals in dimensional regularization made simple, Phys. Rev. Lett. 110 (2013) 251601 [arXiv: 1304.1806] [INSPIRE].

[126] D. Maître, HPL, a mathematica implementation of the harmonic polylogarithms, Comput. Phys. Commun. 174 (2006) 222 [hep-ph/0507152] [INSPIRE].

[127] A. Brandhuber, M. Kostacinska, B. Penante and G. Travaglini, $\operatorname{Tr}\left(F^{3}\right)$ supersymmetric form factors and maximal transcendentality Part I: $\mathcal{N}=4$ super Yang-Mills, JHEP 12 (2018) 076 [arXiv: 1804.05703] [INSPIRE].

[128] A. Brandhuber, M. Kostacinska, B. Penante and G. Travaglini, $\operatorname{Tr}\left(F^{3}\right)$ supersymmetric form factors and maximal transcendentality Part II: $0<\mathcal{N}<4$ super Yang-Mills, JHEP 12 (2018) 077 [arXiv: 1804.05828] [INSPIRE].

[129] M. Assadsolimani, S. Becker and S. Weinzierl, A Simple formula for the infrared singular part of the integrand of one-loop QCD amplitudes, Phys. Rev. D 81 (2010) 094002 [arXiv:0912.1680] [INSPIRE].

[130] N. Arkani-Hamed, J.L. Bourjaily, F. Cachazo, A.B. Goncharov, A. Postnikov and J. Trnka, Grassmannian Geometry of Scattering Amplitudes, Cambridge University Press (2016) [arXiv:1212.5605] [INSPIRE].

[131] N. Arkani-Hamed, J.L. Bourjaily, F. Cachazo, S. Caron-Huot and J. Trnka, The All-Loop Integrand For Scattering Amplitudes in Planar N=4 SYM, JHEP 01 (2011) 041 [arXiv: 1008.2958] [INSPIRE].

[132] N. Arkani-Hamed, J.L. Bourjaily, F. Cachazo and J. Trnka, Local Integrals for Planar Scattering Amplitudes, JHEP 06 (2012) 125 [arXiv:1012.6032] [INSPIRE].

[133] J.L. Bourjaily and J. Trnka, Local Integrand Representations of All Two-Loop Amplitudes in Planar SYM, JHEP 08 (2015) 119 [arXiv:1505.05886] [INSPIRE].

[134] Z. Bern, E. Herrmann, S. Litsey, J. Stankowicz and J. Trnka, Evidence for a Nonplanar Amplituhedron, JHEP 06 (2016) 098 [arXiv:1512.08591] [INSPIRE].

[135] J.L. Bourjaily, E. Herrmann, C. Langer, A.J. McLeod and J. Trnka, Prescriptive Unitarity for Non-Planar Six-Particle Amplitudes at Two Loops, JHEP 12 (2019) 073 [arXiv: 1909.09131] [INSPIRE].

[136] J.L. Bourjaily, E. Herrmann, C. Langer, A.J. McLeod and J. Trnka, All-Multiplicity Non-Planar MHV Amplitudes in SYM at Two Loops, arXiv:1911.09106 [INSPIRE].

[137] S. Badger et al., Applications of integrand reduction to two-loop five-point scattering amplitudes in QCD, PoS (LL2018) 006 (2018) [arXiv: 1807.09709] [INSPIRE].

[138] S. Abreu, F. Febres Cordero, H. Ita, B. Page and V. Sotnikov, Planar Two-Loop Five-Parton Amplitudes from Numerical Unitarity, JHEP 11 (2018) 116 [arXiv: 1809.09067] [INSPIRE]. 
[139] S. Abreu, J. Dormans, F. Febres Cordero, H. Ita, B. Page and V. Sotnikov, Analytic Form of the Planar Two-Loop Five-Parton Scattering Amplitudes in QCD, JHEP 05 (2019) 084 [arXiv: 1904.00945] [INSPIRE].

[140] S. Badger et al., Analytic form of the full two-loop five-gluon all-plus helicity amplitude, Phys. Rev. Lett. 123 (2019) 071601 [arXiv: 1905.03733] [INSPIRE].

[141] T. Ahmed, J. Henn and B. Mistlberger, Four-particle scattering amplitudes in QCD at NNLO to higher orders in the dimensional regulator, JHEP 12 (2019) 177 [arXiv: 1910.06684] [INSPIRE].

[142] V. Del Duca, C. Duhr, R. Marzucca and B. Verbeek, The analytic structure and the transcendental weight of the BFKL ladder at NLL accuracy, JHEP 10 (2017) 001 [arXiv: 1705.10163] [INSPIRE].

[143] W.L. van Neerven, Infrared Behavior of On-shell Form-factors in a $N=4$ Supersymmetric Yang-Mills Field Theory, Z. Phys. C 30 (1986) 595 [InSPIRE].

[144] T. Gehrmann, J.M. Henn and T. Huber, The three-loop form factor in $N=4$ super Yang-Mills, JHEP 03 (2012) 101 [arXiv:1112.4524] [INSPIRE].

[145] A. Brandhuber, G. Travaglini and G. Yang, Analytic two-loop form factors in $N=4 S Y M$, JHEP 05 (2012) 082 [arXiv:1201.4170] [InSPIRE].

[146] K.S. Kolbig, Nielsen's generalized polylogarithms, SIAM J. Math. Anal. 17 (1986) 1232 [INSPIRE]. 